CAAC CUENCA

Colecciones y Archivos de Arte Contemporáneo de Cuenca

CAAC CUENCA

Contemporary Art Archives and Collections of Cuenca

José Ramón Alcalá \& Vicente Jarque

Cuenca: desde el puente de los años Cuenca: across the bridge of years

\title{
Francisco Jarauta
}

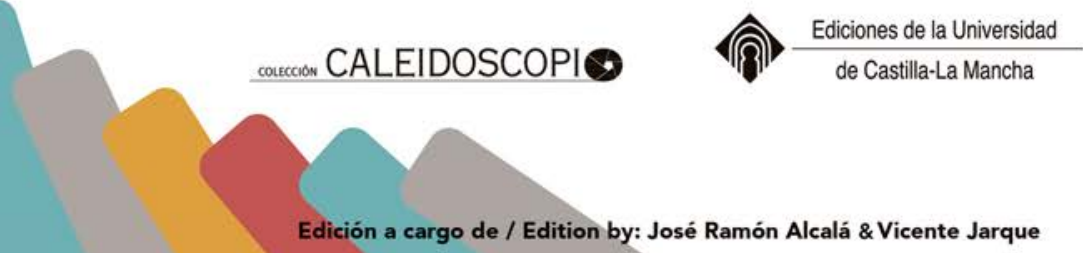


Inauguración de la exposición "Colecciones y Archivos de Arte Contemporáneo" organizada por los miembros coordinadores de CAAC para su presentación, por primera vez, al público. Facultad de Bellas Artes. Cuenca. Noviembre 2013 / Opening of the exhibition "Collections and Archives of Contemporary Art" organized by the coordinators members of CAAC for the first time presentation to the public. School of Fine Arts. Cuenca. November 2013

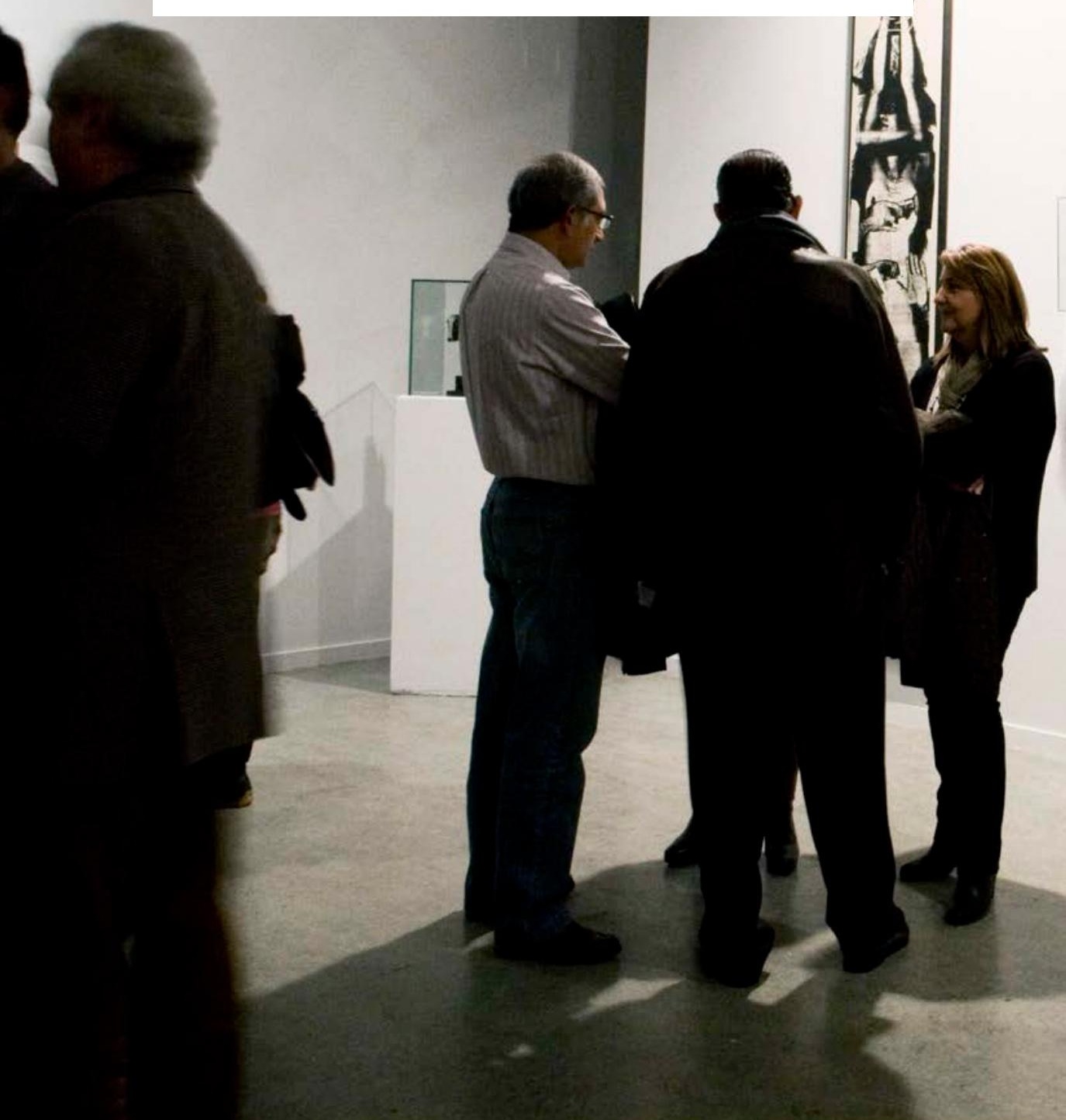




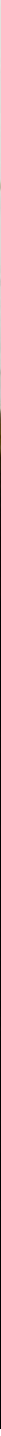


Inauguración de la exposición "Colecciones y Archivos de Arte Contemporáneo" organizada por los miembros coordinadores de CAAC para su presentación, por primera vez, al público. Facultad de Bellas Artes. Cuenca. Noviembre 2013 / Opening of the exhibition "Collections and Archives of Contemporary Art" organized by the coordinators members of CAAC for the first time presentation to the public. School of Fine Arts. Cuenca. November 2013

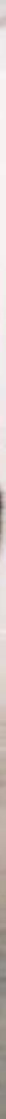



La Facultad de Bellas Artes de la Universidad de Castilla-La Mancha (UCLM) cumple este 2016 treinta años de existencia, solo unos pocos menos que la misma Universidad que, desde sus orígenes, ha jugado un papel esencial en la conformación de un espacio autonómico y de una identidad social que, a día de hoy, resurge como una espléndida realidad llena de ilusión por su futuro.

Del mismo modo que la UCLM ha hecho región, nuestra Facultad de Bellas Artes ha hecho esto mismo y mucho más en el ámbito de las artes, las prácticas artísticas y la cultura en general, tanto para la ciudad de Cuenca -donde radica su sede- como para esta región castellano-manchega y el conjunto del panorama nacional e internacional, pues desde sus orígenes supuso un revulsivo que removió el espíritu crítico y cultural de la sociedad de su entorno natural, posicionándose como una de las Facultades en Bellas Artes de vanguardia, tanto en el plano educativo como en el de la creación y la investigación de las prácticas artísticas contemporáneas.

El conjunto del profesorado y estudiantes que han conformado y configurado como una brillante realidad el presente de nuestra $\mathrm{Fa}-$ cultad, con su trabajo y estudios durante estos treinta años, se plasma a día de hoy en la existencia de un conjunto de archivos de investigación y colecciones artísticas, que nos perfilan con una identidad singular dentro de España por el magnífico patrimonio artístico y cultural que atesoramos. Es por el trabajo de estos profesores, y de sus estudiantes y gestores, por lo que la Facultad de Bellas Artes conserva y mantiene una riqueza patrimonial que hoy quiere poner en valor y presentar a la sociedad, para que revierta en su beneficio y, revisando este periodo, podamos obtener una visión global de lo que ha supuesto nuestro pasado más reciente en lo tocante al arte contemporáneo. 
Por este motivo, desde la Facultad se decidió crear las CAAC, Colecciones y Archivos de Arte Contemporáneo, que se presenta aunando este conjunto de esfuerzos y voluntades creativas existentes: el Archivo Virtual de Artes Escénicas, el Centro de Creación Experimental, Acción GME 2.4, Medios de Impresión Bellas Artes UCLM Cuenca, MIDECIANT, Fondos de Obra Gráfica de la Galería Juana Mordó, la colección Parkett y el Archivo de Documentación Pedro Almodóvar.

Desde el Vicerrectorado de Cultura, Deporte y Extensión Universitaria de la UCLM estamos convencidos de la importancia que poseen las CAAC tanto para nuestra universidad como para la Facultad, la ciudad de Cuenca, la región de Castilla-La Mancha y, por supuesto, para todo el mundo, motivo por el cual apoyamos desde sus orígenes las iniciativas generadas desde la facultad de Bellas Artes para poner en valor el conjunto de estas colecciones, a partir de un trabajo intenso, minucioso, profesional y global, para conocer en detalle la riqueza patrimonial y cultural que contienen, y que ahora queremos dar a conocer, con el mismo orgullo con el que nacimos: la conciencia de que nuestro principal motivo de existencia son nuestros estudiantes, nuestros profesionales y nuestra sociedad, que están participando también desde sus orígenes, en este apasionante proceso de creación, promoción y difusión del conocimiento en las prácticas artísticas contemporáneas. 
El libro que ahora ponemos a disposición del público interesado no es más que una muestra de lo que somos como Universidad de Castilla-La Mancha, desde lo que hemos sido y pretendemos ser como Facultad de Bellas Artes, y de lo como Vicerrectorado de Cultura, Deporte y Extensión Universitaria que consideramos que debe ser nuestro papel motor y dinamizador de esta parcela crucial del Saber en la sociedad actual. Desde aquí, nuestra más sincera enhorabuena a todos cuantos han hecho posible la existencia de estas colecciones en nuestra Facultad, y a todos cuantos están trabajando en torno a ella para hacer de las CAAC un centro de referencia -que lo es- en el panorama artístico nacional e internacional.

Maria de los Ángeles Zurilla

Vicerrectora de Cultura, Deporte y Extensión Universitaria

Universidad de Castilla-la Mancha 
In 2016, the School of Fine Arts at the University of Castilla-La Mancha (UCLM) celebrates its 30-year anniversary, just a few years less than the University itself, which has played an essential role since the very beginning in creating an independent space and a social identity which has emerged as a splendid reality full of illusion for the future.

Just as UCLM has carved out its own space, our School of Fine Arts has followed a similar path, and gone even further in the field of the arts, artistic practices, and culture in general. Its efforts are evident not only in the city of Cuenca - where it is located - but in all of Castilla-La Mancha, throughout Spain and the world. Since its creation, it has had a positive effect, stirring society's critical and cultural spirit in this natural setting, positioning itself as a state-of-the-art School of Fine Arts, not only in an educational sense, but creatively and in research related to contemporary artistic practices.

The teaching staff and student body who have given shape to the brilliant reality which is the School we see today, with their hard work and studies over these past thirty years, are now embodied in the existence of a set of research and artistic collection archives. They lend us a unique identity in Spain thanks to the magnificent artistic and cultural heritage found here. It is because of the work of these professors, and their students and administrators, because of the rich legacy conserved and maintained by the School of Fine Arts, whose value it now wishes to highlight and present to society so that it can reap the benefits and review this period of history, that we are afforded a global vision of what our recent past has meant within the context of contemporary art. 
It is for this reason that the School of Art has decided to create the CAAC - the Center for Contemporary Art Archives and Collections - which is presented in a coordination of the existing efforts and creative will of the following: the Virtual Performing Arts Archive, the Center for Experimental Creation, the GME 2.4 Action, Fine Arts Print Media UCLM Cuenca, MIDECIANT, the Graphic Arts Collection from the Juana Mordó Gallery, the Parkett Collection and the Pedro Almodóvar Archive.

At the office of the Assistant Dean for Culture, Sports and University Extension Courses at UCLM, we are convinced of the importance of the CAAC not only for our university, but for the School of Fine Arts, the city of Cuenca, the region of Castilla-La Mancha, and of course, for the entire world. That is why from the very beginning we have supported the initiatives at the School of Fine Arts for underlining the collective value of these collections. The intense, detailed, professional and global work of many has enabled us to learn about the cultural and patrimonial value within, which we are now in a position to share, with the same pride we felt when our school opened: the awareness that our main motive for being is our students, our professionals and our society, all of whom have participated from the beginning in this incredible process of creation, promotion and dissemination of the knowledge of contemporary artistic practices. 
The book which we are now making available to the public is nothing more than a sample of who we are at the University of Castilla-La Mancha, of who we have been and who we aim to be as a School of Fine Arts, and what the Assistant Dean of Culture, Sports and University Extension Courses considers to be our driving role and catalyst, one as crucial as Wisdom in our current society. From the office of the Assistant Dean, we extend our most sincere congratulations to all those who have made possible the existence of these collections in our School, and to all those who have worked to make the CAAC a center of reference - and it is - on the artistic landscape of Spain and the international art world.

María de los Ángeles Zurilla

Assistant Dean of Culture, Sports and University Extension Courses University of Castilla-La Mancha 

Exposición "Colecciones y Archivos de Arte Contemporáneo" organizada por los miembros coordinadores de CAAC para su presentación, por primera vez, al público. Facultad de Bellas Artes. Cuenca. Noviembre 2013 / Exhibition "Collections and Archives of Contemporary Art" organized by the coordinators members of CAAC for the first time presentation to the public. School of Fine Arts. Cuenca. November 2013

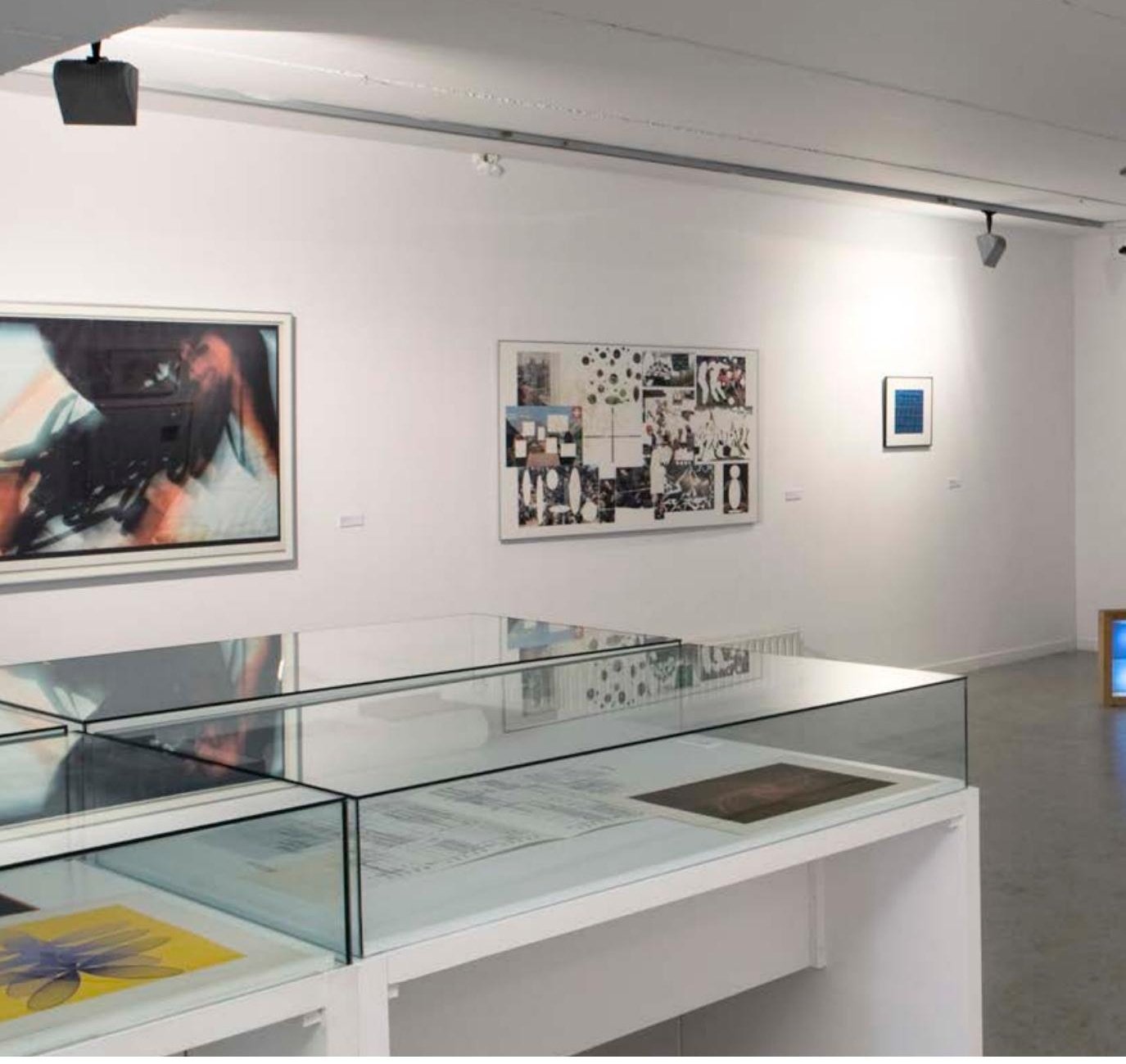



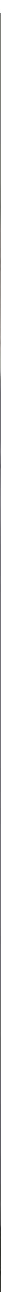
Exposición "Colecciones y Archivos de Arte Contemporáneo" organizada por los miembros coordinadores de CAAC para su presentación, por primera vez, al público. Facultad de Bellas Artes. Cuenca. Noviembre 2013 / Exhibition "Collections and Archives of Contemporary Art" organized by the coordinators members of CAAC for the first time presentation to the public. School of Fine Arts. Cuenca. November 2013
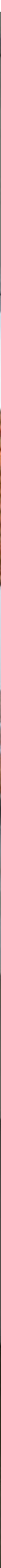


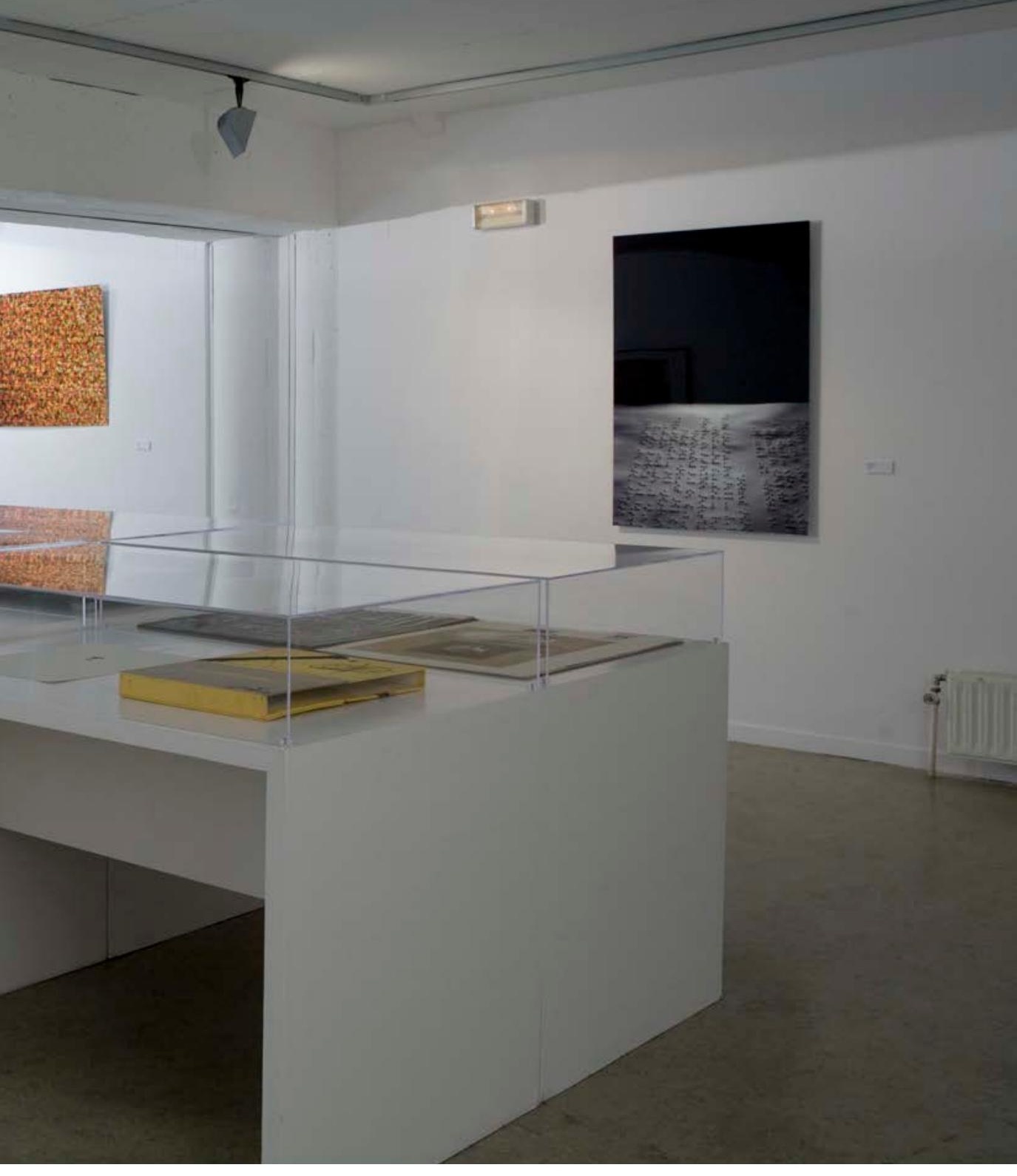


Desde su creación, la Facultad de Bellas Artes de Cuenca se viene distinguiendo en el ámbito de la enseñanza artística superior por su marcado carácter innovador, manteniendo una firme apuesta por la transversalidad y la descategorización de los diferentes medios artísticos. La formación que se oferta desde la Facultad y la selección de su profesorado han sido concebidas desde el inicio atendiendo a la convicción de que la tradición del arte solo puede transmitirse con éxito en el actual contexto histórico, vertiginoso y lleno de incertidumbres, mediante la permanente conexión con las nuevas ideas y prácticas auspiciadas por un desarrollo tecnológico que está transformando a pasos agigantados nuestras sociedades.

Resultado de esta perspectiva, a lo largo de los tres últimos decenios, gracias a importantes donaciones y al fruto del trabajo de los diferentes grupos de investigación con presencia en la Facultad, se ha logrado reunir un conjunto de colecciones de obras y documentos que, no por azar, vienen a cubrir los más diversos ámbitos de la producción artística contemporánea. El patrimonio así acumulado constituye un valioso instrumento de trabajo no solo desde el punto de vista de la investigación, sino también de la docencia.

La Facultad de Bellas Artes no aspira, por supuesto, a erigirse en museo, pero sí a seguir cuidando de sus colecciones, a ampliarlas y, desde luego, a abrirlas a la ciudad que la acoge. Este es el espíritu con el que han nacido las Colecciones y Archivos de Arte Contemporáneo que ahora se presentan al público. En este sentido, el catálogo que ahora se edita puede ser entendido como la memoria de un trabajo realizado, pero también como testimonio de un presente y de la esperanza en un futuro en el que nuestra Facultad habrá de seguir desempeñando una relevante contribución en el desarrollo del arte y la cultura en Castilla-La Mancha.

\section{Javier Diez de Baldeón}

Decano de la Facultad de Bellas Artes 
Since its creation the Faculty of Fine Arts of Cuenca has been distinguishing in the field of higher art education for its strong innovative character, maintaining a firm commitment to mainstreaming and decategorisation of different artistic media. The training is offered by the Faculty and the selection of its teaching staff have been conceived from the start in response to the conviction that the tradition of art can only be transmitted successfully in the current historical, giddy and full of uncertainties context, through permanent connection with new ideas and practices sponsored by a technological development that is transforming our societies leaps and bounds.

The outcome of this perspective, over the last three decades, due to important donations and the result of the work of the different research groups with a presence in the Faculty, it has been managed to bring together a set of artworks's collections and documents that, not by chance, come to cover the most diverse spheres of contemporary artistic production. The accumulated heritage is a valuable working tool not only from the point of view of research, but also teaching.

The Faculty of Fine Arts does not aspire, of course, to establish itself in museum but it does continue to care for its collections, expand and, also, open them to the city that welcomes it. This is the spirit with which Collections and Archives of Contemporary Art were born, now presented to the public. In this sense, the catalog that are now published can be understood as the memory of a work, but also as testimony of the present and the hope for a future in which our faculty will continue to play a relevant contribution to the development of the art and culture in Castilla-La Mancha.

Javier Diez de Baldeón

Dean of The Faculty of Fine Arts 


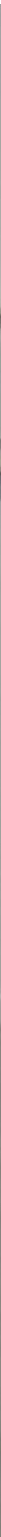


Exposición "Nuevos planteamientos de investigación en las CAAC. Pasado, presente y futuro" realizada con motivo de las primeras jornadas oficiales de las CAAC.

Comisarias: Beatriz Escribano y Cristina Peña. Facultad de Bellas Artes. Cuenca. Abril 2016 / Exhibition "New research approaches in the CAAC. Past, present and future" made on the occasion of the first official conference of the CAAC. Curators: Beatriz Escribano y Cristina Peña. School of Fine Arts. Cuenca. April 2016

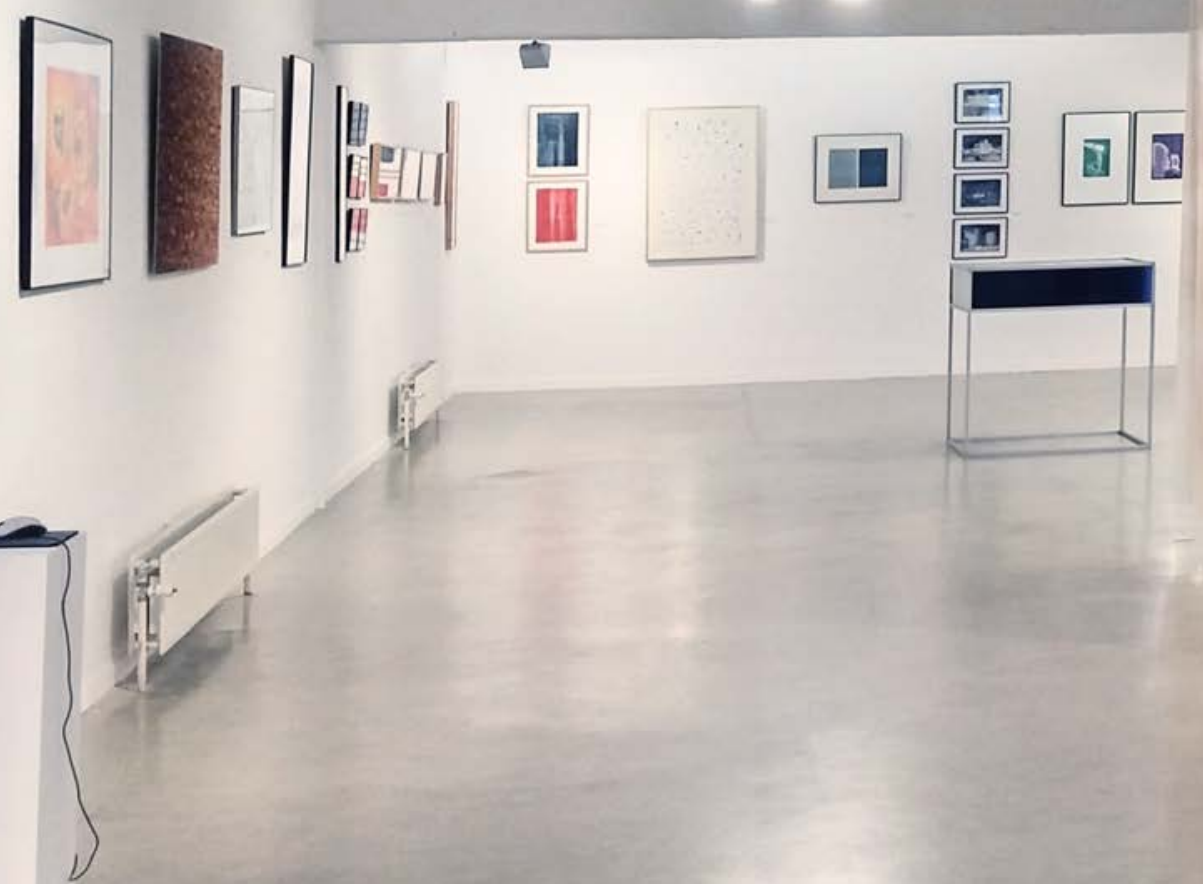




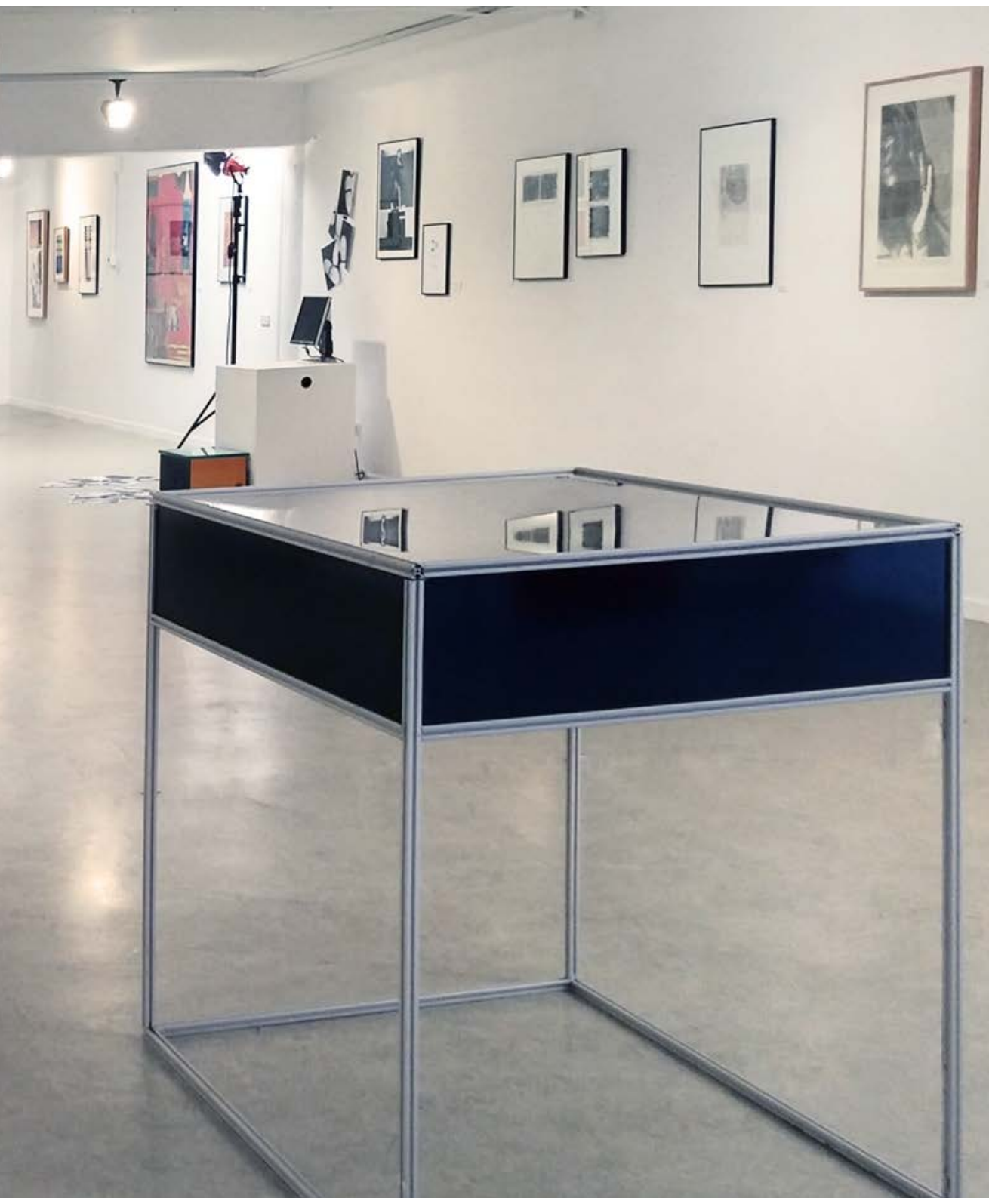





\section{CAAC CUENCA \\ Colecciones y Archivos de Arte Contemporáneo de Cuenca.}

Todos sabemos lo que es una ciudad. O mejor: lo que no es. Una ciudad no es una mera acumulación de edificios determinados por la división del trabajo (viviendas, servicios, oficinas, centros de ocio), conectados por las correspondientes vías de comunicación (calles, plazas, con algún parque o jardín intercalado) y rodeados por polígonos industriales y urbanizaciones de uso residencial. Una ciudad solo puede ser eso en la medida en que sus habitantes la hacen suya e invisten a todos esos elementos de alguna forma de significación que articule y confiera un sentido profundo a la convivencia humana. En la medida en que los significados puedan ser compartidos y reconocidos desde fuera, la ciudad adquiere esa dimensión simbólica de la que procede la que será su identidad y, por tanto, su diferencia.

Una ciudad como Cuenca, en cuyo lema promocional se nos dice que es "única" (como todas, al fin y al cabo), tiene que afirmar su identidad con arreglo a sus cualidades específicas. Estas tienen que ver ante todo, sin duda, con su extraordinario casco antiguo, encaramado entre las hoces de los ríos Júcar y Huécar (con esa singular abundancia de rincones, recovecos, cuestas y piedras nobles), así como con la inmediata presencia de la naturaleza, con la que uno se da de bruces desde las terrazas traseras de las casas de las calles que suben hasta el Barrio del Castillo (como desde la que fue casa de Antonio Saura, quien quedó fascinado por los "ojos de la mora", esas dos cavidades que resaltan entre las peñas de la Hoz del Júcar). Todo esto, unido a la afabilidad de sus barrios típicos, al bullicio del downtown -junto a las fiestas y tradiciones, claro está- constituye el conjunto de los escenarios en donde germinan los sentidos de pertenencia entre sus habitantes. 
Pero todo esto lo ha tenido Cuenca desde hace muchos años, desde antes de que las ciudades - tanto las grandes como las pequeñas, cada una a su manera- se vieran arrastradas por un universo histórico caracterizado por el progresivo reconocimiento de la diversidad cultural, la globalización de las conciencias y el irresistible predominio de las tecnologías. El capital simbólico de las ciudades ha de adaptarse a la actual sociedad del conocimiento y no puede, por tanto, sino abrirse y disponerse a una permanente asunción de lo nuevo, a la recepción de formas siempre cambiantes de experiencia y a su adecuada asimilación en términos de enriquecimiento y de transformación de las identidades heredadas.

Es en este punto en el que, al lado de las oportunas iniciativas de orden económico y las políticas de cohesión social, ha de intervenir eso que llamamos cultura. De hecho, es la cultura, entendida en su sentido fuerte, no solo como el conjunto de las formas de vida social que hace humano al ser humano en general, sino en su acepción más estricta en cuanto que producto de una orientación concreta de la conciencia, la sensibilidad y del intelecto, la que termina por erigirse como la puerta principal a través de la cual puede una ciudad ingresar de verdad en el mundo contemporáneo. Es la cultura la que, a fin de cuentas, puede articular en términos cualitativos la identidad ciudadana y, trascendiendo sus límites, proyectar su imagen hacia el exterior. Y es también la que recoge la realidad histórica y establece el campo de juego en donde puede construirse el presente de una ciudad con futuro.

Y lo cierto es que, a este respecto, cabe decir de la ciudad de Cuenca ha resultado ser bastante afortunada. Se cuenta que, en una noche de verano de 1963, seguramente atraídos por el encanto de su casco viejo, a un grupo de artistas reunidos en torno a Gustavo Torner, entre los que se encontraban Eusebio Sempere y Fernando Zóbel, compartieron una cena que resultaría decisiva. Zóbel había reunido una considerable colección de arte abstracto, al que él mismo se dedi- 
caba, y buscaba una sede en donde exponerlo. Cuando se supo de la posibilidad de rehabilitar y ocupar las Casas Colgadas, rescatándolas del largo sueño medieval, la suerte quedó echada. Tres años después abriría sus puertas el Museo de Arte Abstracto Español, un centro por entonces absolutamente "único", en donde se acogía una forma de pintura hecha en nuestro país que, por vez primera desde la Guerra Civil, estaba a la altura de la conciencia alcanzada por el arte a nivel internacional. Las obras autores ya por entonces tan significativos como Zóbel, Torner, Rueda, Rivera, Sempere, Millares, Saura, Canogar, Yturralde, Palazuelo, Feito, Viola, Chillida, Tàpies, entre otros, hallaron en Cuenca un acomodo insospechado y, como luego se vería, incontestablemente fructífero.

Otro personaje fundamental para entender la actual posición de Cuenca como foco de producción e irradiación de cultura ha sido y sigue siendo, por supuesto, Antonio Pérez. Hombre inquieto y viajero, amante del arte y de los libros, remontador de ríos, un buen día de 1957 llegó hasta Cuenca, conoció a Millares y a Saura y, tras sus largos años pasados en París, en donde participaría de la fundación, junto a José Martínez, de la legendaria editorial El Ruedo Ibérico, a su regreso también se instalaría aquí, también en el barrio alto, precisamente frente al caserón de Saura. Por allí pasarían artistas y escritores, gentes como sus amigos Juan Marsé o Ramón Chao, así como figuras de la altura de Alejo Carpentier, Julio Cortázar, Cabrera Infante o Roberto Bolaño, entre tantos otros. Su pasión de lector y editor, de coleccionista y de artista de los objetos encontrados, llevaría con el tiempo, en 1997, a la inauguración de la Fundación Antonio Pérez, en donde la tradición representada en el Museo de Arte Abstracto se prolonga, en forma de una colección en permanente crecimiento y de numerosas exposiciones temporales, hasta el arte de nuestros días. 
A todo esto hay que añadir el privilegio del que ha gozado Cuenca de albergar, desde la segunda mitad del siglo pasado, la sede del Festival de Música Religiosa, un evento de innegable reconocimiento internacional, así como la creación del Gabinete de Música Electrónica, inaugurado en el Conservatorio de Música, en ambos casos bajo la dirección de Pablo López de Osaba. En sus instalaciones trabajaron -bajo el asesoramiento del técnico Leopoldo Amigo, en una primera etapa, y posteriormente del compositor electroacústico Julio Sanz- músicos y compositores experimentales de la talla del chileno Gabriel Brncic (la figura fundamental), Francisco Kröpf, Adolfo Núñez, Eva Lootz, José Iges, Concha Jerez, Jesús Rueda, Gregorio Jiménez, José Manuel Berenguer, Mauricio Soteloy o Eduardo Polonio. En esta misma línea, y como continuación de aquellos esfuerzos pioneros, los Seminarios Internacionales sobre Música y Ordenadores en la Universidad Internacional Menéndez y Pelayo, bajo la atenta mirada y con la cooperación y seguimiento de la asociación AVADI, han venido contribuyendo a la difusión, desarrollo y conservación del ya importante legado de la música electroacústica en Cuenca, algo de lo que desde principios del 2014 se ocupa la asociación Acción GMEv2.4.

Finalmente, en cuanto a la Fundación Antonio Saura, inaugurada a principios de siglo en la Casa Zavala, con independencia de la triste polémica que tuvo lugar a propósito de las últimas voluntades del artista y de sus eventuales dificultades relativas a una clara definición de su línea expositiva, puede considerarse igualmente como una propuesta directamente enlazada con las anteriores iniciativas, todas ellas asociadas al propósito de la inserción del arte contemporáneo en la ciudad de Cuenca (así como de Cuenca en el marco de la cultura contemporánea) y más o menos armónicamente dirigidas, por tanto, hacia la configuración de un contexto singular sin el cual sería difícil de explicar la existencia del proyecto que aquí presentamos. 


\section{La creación de la Facultad de Bellas Artes}

En efecto, siguiendo este camino de apuesta por la cultura en y desde Cuenca, y evidentemente al hilo de todos estos precedentes que se iban encadenando, en 1985 se tomó la decisión política de ubicar en esta ciudad la Facultad de Bellas Artes de la recién creada Universidad de Castilla-La Mancha. Se trataba de un proyecto pionero y original cuya concepción y puesta en funcionamiento fueron encargados, como un experimento académico, a algunos viejos participantes del grupo El Paso, todos ellos muy vinculados emocionalmente, por las razones que antes hemos comentado, al proyecto artístico-cultural de la ciudad conquense. Estos, en segunda instancia, delegaron la tarea docente a un grupo de jóvenes artistas, reconocidos practicantes de diferentes movimientos del arte de vanguardia de la segunda mitad del siglo XX. La selección de los futuros docentes la realizaron miembros del citado grupo, tras varias convocatorias públicas que se propusieron durante los primeros años fundacionales. Así es como fueron contratados, entre otros, José Antonio Sarmiento, Antonio Cano, José Luis Brea, José Ramón Alcalá, Horacio Fernández, y también artistas de prestigio como Eva Lootz, Gonzalo Puch, Jaime Lorente o Ricardo Cadenas, todos ellos acreditados por una incipiente pero ya consolidada trayectoria teórica y/o artística, a los cuales se fueron añadiendo con el tiempo otros nombres igualmente cualificados y concordantes con esa misma línea de innovación que habría de caracterizar hasta el presente el espíritu de la Facultad.

Una de las consecuencias principales de esta apuesta por el futuro y la orientación de la docencia más allá de los estrechos márgenes de la vida académica tradicional fue la creación, por parte de estos jóvenes artistas-profesores, de diversos programas de investigación vinculados a los nuevos movimientos artísticos de vanguardia. También se pusieron en funcionamiento centros de creación e investigación pioneros en su especie, como el Museo Internacional de Electrografía (el 
MIDE, que desde sus mismos comienzos sería, además, Centro de Innovación en Arte y Nuevas Tecnologías), el Centro de Creación Experimental (que se especializó en la investigación, creación y producción de arte sonoro y performativo) y, años más tarde, el Instituto de Creación Audiovisual y el Proyecto de Artes Escénicas.

Asimismo, la Facultad de Bellas Artes organizó el histórico Congreso La Situación, al que asistirían muchos de los más relevantes artistas jóvenes de la escena nacional, y que más tarde daría lugar a las varias ediciones del festival de prácticas artísticas de vanguardia Situaciones. Con inhabitual rapidez, toda esta producción de arte de vanguardia y su consecuente repercusión en sus aulas convirtió al centro en referencia nacional e internacional. Sirvió de impulso motor para la renovación de los Planes de Estudios y de las estrategias metodológicas de muchas de las facultades de bellas artes españolas, aún ancladas por entonces en concepciones tradicionales pedagogías vetustas. La Facultad de Bellas Artes de la UCLM representó, además, un modelo para la creación de otras facultades en el territorio tanto nacional como internacional.

\section{Crisis y resistencias culturales: creación de las CAAC}

A finales de la primera década de este siglo, los efectos perversos de la brutal crisis económica que comenzó a azotar el país también se dejaron sentir, como es bien sabido, en el campo cultural. Incluso, si cabe, con mayor intensidad que en otros campos al cuidado de la Administración, hasta el punto de poner en serio peligro la continuidad de muchas de las iniciativas de las que venimos hablando. Desde entonces, por ejemplo, el Gabinete de Música Electroacústica se encuentra cerrado y su maquinaria sonora paralizada; el Museo Internacional de Electrografía tuvo que cerrar sus talleres y laboratorios y ceder sus equipos a la Facultad de Bellas Artes, así como trasvasar sus fondos bibliográficos y su mediateca a la Biblioteca General del 
Campus Universitario; mientras tanto, la Fundación Antonio Saura se ve enfrentada a problemas financieros que podrían amenazar seriamente su continuidad a corto plazo.

En este contexto de retroceso de políticas culturales estratégicas, no solo en el ámbito nacional sino regional y local, a finales de 2013 distintos profesores de la Facultad de Bellas Artes se reunieron con el objetivo principal de revisar en profundidad el alcance y naturaleza de sus trabajos y actividades. Se trataba de galvanizar esas corrientes culturales que dan un sentido identitario y de pertenencia a la ciudad, esta vez en un contexto de crisis económica y social. Urgía repensar las funciones de las colecciones de la Facultad de Bellas Artes y articularlas en el marco de los museos de la ciudad como parte de una estrategia de recuperación y puesta en valor de un patrimonio artístico, en primera instancia, y de la propia ciudad de Cuenca, en definitiva, como centro cultural.

Una de las primeras decisiones fundamentales que se tomó fue la de reunir todo el trabajo realizado y las obras de vanguardia acumuladas para ponerlo todo en valor de forma conjunta y así poder darlo a conocer y permitir el acceso público a sus fondos permanentes. Se trata de fortalecer los lazos de interdependencia entre obras radicadas en Cuenca y los circuitos de discusión artística internacionales. La consecuencia de estas reuniones fue la creación y puesta en funcionamiento, en una primera instancia, de las Colecciones y Archivos de Arte Contemporáneo (CAAC_Cuenca) de la Facultad de Bellas Artes de Cuenca (www.caac.uclm.es). El proyecto partía del ejercicio de memoria colectiva que permitía el inventario de los bienes artísticos producidos y atesorados, para proceder a su correcta organización y clasificación. Los primeros resultados, obtenidos a mediados del año 2014, mostraban un valioso conjunto de obras, piezas, archivos y documentos relacionado con las prácticas artísticas de la vanguardia reciente. 
La fortaleza más significativa de este conjunto de colecciones era la radicalidad y el carácter innovador de sus contenidos. Las prácticas más vanguardistas habían conducido a sus artistas a producir en un contexto alternativo y de orientación contra-cultural refractario al circuito tradicional del museo-galería de arte. El carácter experimental y transgresor de las obras ha producido la curiosa situación de que, a pesar de la repercusión mediática que todas estas prácticas habían generado, resulta muy complicado en la actualidad encontrarlas entre las numerosas colecciones de arte contemporáneo que se han ido construyendo durante la segunda mitad del siglo pasado y comienzos del presente. En este contexto, los fondos permanentes de las CAAC_Cuenca centran su atención y actividad en facilitar el acceso y la divulgación de estas obras.

\section{Archivos y colecciones}

Estos fondos han sido reunidos, a lo largo de los últimos veinticinco años, al hilo de las diversas circunstancias que se explican en los textos introductorios a cada una de las secciones que componen el catálogo de las CAAC_Cuenca. Las colecciones del MIDE comenzaron como un esfuerzo sin precedentes en la producción experimental de obras de copy-art; con el tiempo, sin embargo, este centro de investigación se ha ido convirtiendo -más allá de los registros museísticos habituales- en un lugar no solo de conservación, sino de producción de trabajos vinculados a las nuevas tecnologías de la imagen digital y del arte multimedia. Su carácter eminentemente experimental, junto con su permanente atención a los nuevos medios, es igualmente compartido por la labor del Gabinete de Música Electroacústica (GME), el cual, tras los avatares de los que ya hemos hablado anteriormente, se muestra revitalizado gracias a la iniciativa de nuestros docentes. Lo mismo cabe decir del Archivo Virtual de Artes Escénicas (AVAE). Este, centrado en la escena de vanguardia, permite incluir entre su 
campo de trabajo no solo el teatro y la danza contemporáneos, sino, asimismo acciones, instalaciones, vídeos o performances, junto con el pertinente material teórico, cuenta con un equipo de investigadores presente en distintas universidades españolas y latinoamericanas. La Colección de Medios de Impresión (CMI) de la Facultad se define como un archivo en el que se han venido incorporando año tras año los mejores trabajos de estampa realizados por los estudiantes, y algún profesor, en aras de ofrecer el que ya es un muy amplio panorama de la manera en que han ido evolucionando estas prácticas a desde finales del siglo pasado. En cuanto a los archivos históricos del portal Aleph-Arts.org, se trata de una de las primeras recopilaciones de net-art, que fue concebido y diseñado en primera instancia dentro del MIDECIANT por Ricardo Echevarría (entonces becario del museo/centro de investigación) y Luis Fernández (programador), quienes han donado el disco duro que contiene las primeras interfaces electrónicas y la primera documentación generada para este portal antes de la invitación que estos hicieran a José Luis Brea durante su época de profesor de la Facultad de Cuenca, para que se incorporase al proyecto. A todo lo cual hay que añadir el Centro de Creación Experimental (CDCE), en estos momentos dirigido de manera autónoma por José Antonio Sarmiento y que, aun cuando no forme parte de las CAAC, participa plenamente del mismo espíritu que preside sus archivos y colecciones.

Y, finalmente, tres donaciones de un valor indudablemente singular. Por un lado, los Archivos Pedro Almodóvar (Doctor Honoris Causa por la UCLM, como antes lo fue Antonio Saura, a instancias de la Facultad) cedidos por Producciones El Deseo y, por otro, la histórica (aunque todavía en curso) Colección Parkett de arte múltiple y los importantes Fondos de Obra Gráfica de la Galería Juana Mordó, debidas ambas a la particular generosidad de la prestigiosa galerista Helga de Alvear. La primera (solo visible en su conjunto en Zürich y Nueva York, y desde 2010 en Cuenca) se compone de más de dos- 
cientos sesenta múltiples de artistas de primera línea de más de cuarenta países (con nombres como, por ejemplo, Georg Baselitz, Louis Bourgeois, Maurizio Cattelan, Fischli/Weiss, Anish Kapoor, Richard Serra, Rosemarie Trockel, Andy Warhol o Jeff Wall), ofreciendo, por tanto, una riquísima perspectiva de buena parte de lo que ha sido el arte contemporáneo a lo largo de los últimos treinta años. En cuanto a la segunda, con la que contamos desde 2011, consta de cuarenta y dos carpetas de obra gráfica de muchos de los artistas fundamentales que fueron trabajando con la galería Juana Mordó desde 1964 hasta su muerte en 1984, y que su sucesora, Helga de Alvear, siempre interesada en aproximar el arte a la sociedad, ha deseado poner al disposición de los estudiantes y del público en general cediéndolas a la Facultad. $\mathrm{E} 1$ hecho de que entre esos artistas figurasen también los miembros del grupo El Paso y otros abstractos coetáneos nos devuelve, y no por azar, al comienzo de la historia que todavía estamos relatando.

En conjunto, como el lector habrá podido colegir incluso a primera vista, este panorama de archivos y colecciones es testimonio de una actitud y una trayectoria de la Facultad como institución académica, y en concreto de quienes en ella trabajan, claramente determinada por la conciencia del abierto pluralismo que desde hace tiempo viene caracterizando a eso que llamamos arte contemporáneo y que, además de en la pintura y la escultura, el dibujo o el grabado (y la literatura), también se juega su presente y su porvenir en ese abierto campo de juego configurado por los nuevos medios digitales, así como por la fotografía y el vídeo, el cine, las artes escénicas y el nuevo arte sonoro, entre otras infinitas posibilidades. 


\section{Hacia un nuevo centro de arte contemporáneo}

La iniciativa de reunir todos estos componentes tan variados en un solo centro de arte obedece unos propósitos derivados de varias instancias complejas. Ante todo, por supuesto, de lo que se trata es de su puesta en valor y de favorecer una mejor visibilización de los archivos y colecciones de la Facultad en aras de un mejor aprovechamiento de estos por parte de sus estudiantes (y docentes). Junto a esta función pedagógica o didáctica, fundamental en una Facultad de Bellas Artes, se ofrece la perspectiva, igualmente necesaria, de contar con un centro compartido que propicie la investigación y la producción de trabajos orientados en cualquiera de las líneas ya trazadas y, desde luego, de las muchas otras que puedan ir presentándose en el futuro con arreglo a la esencial apertura del que será el Centro de Arte. Archivos y Colecciones (CAAC_Cuenca -las mismas siglas, pero con contenidos y significado ampliados) a toda clase de nuevas propuestas enmarcables en la situación actual de las artes contemporáneas. Finalmente, las tareas de exhibición y divulgación de estos fondos habrían de articularse de cara a una mayor conexión con la vida cultural de la ciudad y con sus específicas necesidades en cuanto a la experiencia del arte.

En esta dirección, quienes formamos parte de este proyecto somos totalmente conscientes de la imposibilidad -o al menos la inoportunidad- de seguir manteniendo sin cambios un modelo de museo, sea este grande o pequeño, heredado de la situación de la cultura europea de hace más de doscientos años, y luego proseguida sin demasiadas transformaciones sustanciales para dar acogida al arte moderno en general y, solo más recientemente, a las distintas formas de arte contemporáneo (fotografía, vídeo, cine, instalaciones, multimedia). La cuestión estriba en aprovechar al máximo la singularidad de nuestros archivos y colecciones (y su constante desarrollo gracias a una ininterrumpida labor de creación y producción) para, sacándolas a la luz, intentar desarrollar en torno a ellas un proceso de interrelación entre el contexto ciudadano y el lugar en que se hallan enclavadas. 
Esta tarea no es fácil. No lo es para ningún centro de arte, cada uno dependiente del alcance de sus específicas fortalezas y debilidades. Pero lo importante, en cualquier caso, es hacer de la necesidad virtud y responder de manera concreta a la situación en que uno se mueve. En lo que respecta al CAAC y a Cuenca, nuestra voluntad tiene que ver con la perspectiva de una integración del centro en el marco de la oferta expositiva y cultural de la ciudad en que nos encontramos, de cuya historia somos deudores. Como se ha indicado al principio de esta presentación, una ciudad no puede construir su identidad sin un conjunto de referencias culturales que sea capaz de hacer suyas; pero un centro cultural como el que aquí se presenta, en tanto que lugar de investigación, producción, exhibición y conservación de obras de arte contemporáneo, no puede sino esforzarse en complementar esas referencias llenando sus lagunas u ofreciéndose como un espacio de experiencia específica. En ello estamos.

José Ramón Alcalá

Vicente Jarque 


\section{CAAC CUENCA \\ Contemporary Art Archives and Collections of Cuenca}

Everyone knows what a city is. Or perhaps we should say: what it is not. A city is not merely a group of buildings classified by a division of labor (dwellings, public services, offices, recreation centers), connected by corresponding transportation routes (streets and plazas, interspersed with parks or gardens) and surrounded by industrial parks and residential housing developments. A city can only be what it is to the extent that its inhabitants make it their own and infuse all of its elements with a meaning that articulates and confers a profound sense of human community. Insofar as its significance can be shared and recognized from the outside, the city takes on the symbolic dimension of what will become its identity, and as such, what will make it stand out from the rest.

A city like Cuenca, whose promotional motto classifies it as "unique" (like all cities, ultimately), must be able to back up its identity on the basis of its specific qualities. First and foremost, these qualities are undoubtedly linked to Cuenca's extraordinary historic district, perched between the Júcar and Huécar river gorges, and their singular abundance of hidden nooks and crannies, hills and great boulders. They are also linked to the overwhelming role played by nature, which is ever-present from the rear terraces of houses lining the streets that lead to the Castle Quarter (like the building that was once home to Antonio Saura, who was so fascinated by the "moor woman's eyes" - two eye-catching hollows nestled among the rocks of the Júcar river gorge). All of this, together with the affability of its traditional neighborhoods, the bustle of downtown, and of course the local festivals and customs, make up the many settings where a sense of belonging can blossom among the city's inhabitants.

But Cuenca has been home to all of these elements for many years, 
long before cities (both large and small, each in its own way) began to be dragged along by a universal history characterized by the progressive acknowledgement of cultural diversity, the globalization of consciousness and the irresistible predominance of technology. The symbolic capital city has had to adapt to the current information society, and therefore has had no choice but to open up and make itself available for the ongoing drive toward the new, for the acceptance of ever-changing experiences, for its sufficient assimilation in enriching terms, and for the transformation of identities passed down from previous generations.

It is on this point, together with opportune initiatives for economic order and policies of social cohesion, where that which we call culture must come into play. In fact, understood in its most potent sense, culture is not merely all of the forms of social life that make human beings human. Rather, it is its strictest acceptance as the product of a specific orientation of the conscience, of sensitivity and of the intellect that eventually becomes the main gate through which a city can truly move into the contemporary world. In the end, it is culture that can use qualitative terms to articulate the identity of its citizens. And by transcending its limitations, it is able to project its own image to the world outside. It is also a collector of historical reality, establishing the playing field where a city with a future can build its presence.

It is fair to say that the city of Cuenca has been extremely lucky. It is said that on a summer night in 1963, a group of artists including Eusebio Sempere and Fernando Zóbel, gathered by Gustavo Torner and probably drawn by the charm of the old town, sat down together for a dinner that would turn out to be momentous.

Zóbel had amassed a considerable collection of abstract art, reflec- 
ting his own field of interest, and he was looking for an exhibition space. When he learned it might be possible to renovate and utilize the Hanging Houses, rescuing them from their long Medieval sleep, the die was cast. Three years later, he would open the doors to the Spanish Abstract Art Museum, a center that was entirely "unique" at the time, a home for the kind of painting undertaken in this country which, for the first time since the Spanish Civil War, was being recognized on an international level. The artists were already quite well known, including such names as Zóbel, Torner, Rueda, Rivera, Sempere, Millares, Saura, Canogar, Yturralde, Palazuelo, Feito, Viola, Chillida, and Tàpies, among others. They were surprised to find how well they would adapt to Cuenca, and later, how unquestionably successful the project would be.

Another figure who is essential for understanding the current position of Cuenca as a focal point for the creation and dissemination of culture, has long been, and clearly continues to be, Antonio Pérez. A restless traveler, lover of art and books, and explorer of rivers, one fine day in 1957 he arrived in Cuenca. He met Millares and Saura, and after many years spent in Paris, where, together with José Martínez, he helped to found the legendary publishing house El Ruedo Ibérico, he returned to settle here, up in the old town, just opposite Saura's noble home. His house would host artists and writers, people like his friends Juan Marsé and Ramón Chao, and such great figures as Alejo Carpentier, Julio Cortázar, Cabrera Infante and Roberto Bolaño, among many others. His passion as a reader and editor, as a collector of and artist working with found objects, would ultimately lead him to open the Antonio Pérez Foundation in 1997. Here, the tradition represented in the Abstract Art Museum is also present in the growing permanent collection, in numerous temporary exhibits, and in the art of our time.

To all of this, we must add that it has been Cuenca's joy and privi- 
lege to host the Religious Music Festival since its inception in the second half of the 20th century, an event of undeniable international renown. The Course on Electronic Music was also created here, at the Conservatory of Music, under the direction of Pablo López de Osaba. There, experimental musicians and composers worked (first under the guidance of technician Leopoldo Amigo, and later of electro-acoustic composer Julio Sanz) of the stature of Chilean Gariel Brncic (the leading figure), Francisco Kröpf, Adolfo Núñez, Eva Lootz, José Iges, Concha Jerez, Jesús Rueda, Gregorio Jiménez, José Manuel Berenguer, Mauricio Soteloy and Eduardo Polonio. Along the same lines, and as a continuation of those pioneering efforts, the International Seminars for Music and Computers were held at Menéndez y Pelayo International University, under the watchful eye, and with the cooperation and monitoring of the AVADI association. The seminars have contributed to the dissemination, development and conservation of the already significant legacy of electro-acoustic music in Cuenca, a subject that has been the focus of the AcciónGMEv2.4 association since early 2014.

Finally, we turn to the Antonio Saura Foundation, which was unveiled in the first years of the 21st century in the Casa Zavala, independently of the unfortunate controversy that took place as a result of the artist's last will and of the difficulties that developed as to the clear definition of the space's expositive focus. The project might be said to be directly linked to previous initiatives, all of which were associated with the goal of bringing contemporary art to the city of Cuenca (as well as bringing Cuenca into the greater framework of contemporary culture). These goals were more or less harmonically directed toward the configuration of a singular context without which it would be difficult to explain the existence of the project presented here.

\section{The Creation of the School of Fine Arts}


Sure enough, by remaining committed to culture in and around Cuenca, and of course following the lead of all these closely linked predecessors, in 1985 the political decision was made to locate the School of Fine Arts for the recently created University of Castilla-La Mancha here in this city. The pioneering original project had been conceived and driven, as an academic experiment, by several former members of the El Paso group, who had been commissioned to do so. All of them had close emotional ties to the artistic-cultural project in the city of Cuenca, for the reasons laid out above. They in turn delegated the task of teaching to a group of young artists, well-known practitioners of different avant-garde art movements from the second half of the 20th century. The selection of the future teachers was carried out by members of El Paso after a series of open calls were held in the first few years after the school was founded. And this how José Antonio Sarmiento, Antonio Cano, José Luis Brea, José Ramón Alcalá, and Horacio Fernández were contracted, as well as artists of such stature as Eva Lootz, Gonzalo Puch, Jaime Lorente and Ricardo Cadenas, among others. All of them were accredited by a nascent but already proven track record in the theoretical and/or art worlds. Over time, they were joined by other equally qualified names, consistent with the same innovative spirit that came to characterize the School, and which continues to do so.

One of the main consequences of this commitment to the future, and the educational point of view that went beyond the narrow margins of traditional academic life, was the creation by these young artist/professors of diverse research programs linked to new avant-garde art movements. Pioneering centers for creation and investigation were also put into operation, like the International Museum of Electrography (or MIDE, which from the very beginning would also be the Center for Innovation in Art and New Technologies), the Center for Experimental Creation (which specialized in the investigation, creation and 
production of sound and performance art), and several years later, the Institute for Audiovisual Creation and the Scenic Arts Project.

Likewise, the School of Fine Arts organized the historic congress The Situation, attended by many of the most relevant young artists on the national scene, which would later lead to the various incarnations of Situations, an avant-garde art festival. Surprisingly quickly, this avant-garde art production and its resulting repercussions in the classrooms turned the center into a reference point both within Spain and around the world. It served as a driving force for the renovation of the school's curriculum and for the methodological strategies of many other Spanish schools of fine arts, which were still mired at the time in ancient traditional concepts and methods. Further, UCLM's School of Fine Arts was a model for the creation of other schools in the field, both nationally and internationally.

\section{Crisis and Cultural Resistance: the Creation of CAAC}

At the end of the first decade of the 21st century, the perverse effects of the brutal economic crisis beginning to afflict the country were also felt, as we well know, in the cultural domain. It may even have had a greater impact than on other fields under the care of the public administration, to the point that many of the above mentioned initiatives were seriously endangered. As a result, for example, the Course on Electronic Music has been inactive since that point, its sound equipment silenced; the International Museum of Electrography was forced to close its workshops and laboratories, and cede its equipment to the School of Fine Arts, as well as transferring its book collections and media library to the main campus library. Meanwhile, the Antonio Saura Foundation is facing financial problems that could seriously threaten its survival in the short term.

Against the backdrop of declining strategic cultural policies, not 
only on a national scale, but regionally and locally, in late 2013 various professors from the School of Fine Arts held a meeting with the main goal of reviewing in depth the scope and character of their work and activity. They hoped to galvanize those cultural currents that give a sense of identity and belonging to the city, this time in the context of an economic and social crisis. It was urgent that they rethink the function of the collections at the School of Fine Arts and articulate them within the framework of the city's museums as part of a recovery strategy and evaluation, firstly, of artistic heritage, and in the long term, for the city of Cuenca itself as a cultural center.

One of the first fundamental decisions made was to gather together all of the work that had been created and the avant-garde pieces that had been collected in order to determine its value as a whole. The permanent collection could thus be promoted and the public could be allowed access. The idea was to strengthen the ties of interdependence between works based in Cuenca and the lines of communication between international artists. The primary result of these meetings was the creation and rollout of the Cuenca Center for Contemporary Art Archives and Collections (CAAC Cuenca) at the School of Fine Arts in Cuenca www.caac.uclm.es. The project sprung from the collective memory that allowed an inventory to be taken of the artistic creations produced and housed here, thus permitting their organization and classification. The initial results, obtained in mid-2014, revealed a valuable collection of works, pieces, archives and documents related to recent avant-garde artistic practices.

The most significant strength of this group of collections was the radical nature and innovative character of the works therein. The most avant-garde practices had driven artists to create in a context that was alternative and oriented toward an impervious counter culture that had little to do with the traditional art museum or gallery. The experimental and transgressive nature of the works has led to 
a curious situation in which, despite widespread media coverage, it is now quite difficult to find them amongst the many collections of contemporary art that have taken shape during the second half of the last century and the first years of the present one. Given this context, CAAC Cuenca has focused its attention and activity on facilitating access to and dissemination of these pieces.

\section{Archives and Collections}

These many collections have been gathered together over the past 25 years through a series of diverse circumstances. All of these are explained in the introductory texts in each of the sections that make up the $\mathrm{CAAC}_{\neg}$ Cuenca catalog. The MIDE collections began with an unprecedented push for the experimental production of Copy Art works. Over time, however, this research center has gone beyond the normal role of a museum to become a space not only for the conservation of, but for the production of works linked to the new technologies of digital imaging and multimedia art. Its imminently experimental character, together with its continual focus on new media, is shared by the Course on Electronic Museum (GME), which, following the events described above, found its second wind thanks to the initiative spearheaded by our teachers. The same can be said of the Virtual Archive of Scenic Arts (AVAE). Focused on the avant-garde scene, the scope of this entity includes not only contemporary theatre and dance, but contemporary initiatives, installations, videos and performance pieces, together with pertinent theoretical materials. Its research team is made up of members from various universities in Spain and Latin America. The Collection of Print Media at the School is defined as an archive which, year after year, has gradually incorporated the finest prints created by students and the occasional professor. Its goal is to continue to offer what is already a vast panorama in keeping with the evolution of these practices since the end of the 20th century. As for the historic archives found at Aleph-Arts. 
org, this is one of the first collections of Net Art. It was conceived of and designed within MIDECIANT by Ricardo Echevarría (while a student at the museum / research center) and Luis Fernández (programmer). They donated the hard drive containing the first electronic interfaces and the first documentation generated by this site before extending an invitation to José Luis Brea while he was a professor at the School in Cuenca, asking him to be part of the project. To all of this, we must add the Center for Experimental Creation (CDCE), currently independently directed by José Antonio Sarmiento. While it does not formally make up part of CAAC, it participates fully in the same spirit that prevails over the CAAC archives and collections.

Finally, three donations of exceptional value. First, the Pedro Almodóvar Archives (named after the renowned director, a recipient of an honorary Doctorate from UCLM, as was Antonio Saura, at the request of the School of Fine Arts) were donated by E1 Deseo Productions. This was followed by the historic (but still current) Parkett Collection of multiple arts, and the important Juana Mordó Gallery Graphic Arts Collection, both due to the incredible generosity of prestigious art collector Helga de Alvear. The Parkett Collection, (exhibited in its entirety only in Zurich and New York, and since 2010 in Cuenca) is made up of more than 260 multiples by top-notch artists from over 40 countries (with names like Georg Baselitz, Louise Bourgeois, Maurizio Cattelan, Fischli/Weiss, Anish Kapoor, Richard Serra, Rosemarie Trockel, Andy Warhol and JeffWall). They were thus able to offer an extremely rich perspective on much of what contemporary art has stood for over the last 30 years. The final collection, which has been in our possession since 2011, consists of 42 print portfolios from many of the key artists who worked with the Juana Mordó Gallery from 1964 until her death in 1984. Her successor, Helga de Alvear, always keen on making art accessible to society, wished to put the works within reach of students and the general public when she donated them to the School. The fact that the El Paso group and certain other 
contemporaries were also included brings us back to the beginning of the story we are still in the process of telling. And that is no accident.

All in all, as readers will have gathered, perhaps even from the start, this range of archives and collections is testimony to an attitude and a track record at the School as an academic institution, and more specifically, to those who work here. These people are clearly driven by an awareness of the open pluralism that has long characterized that which we call contemporary art. It is not only in painting and sculpture, in drawing and printmaking (and literature) that its present and future can be found in that open playing field made up of digital new media, as well as photography and video, cinema, the scenic arts and new sound art, among other endless possibilities.

\section{Toward a New Center for Contemporary Art}

The initiative to group all of these extremely varied components together in a single art center is in keeping with several objectives derived from various complex instances. Above all else, of course, it has to do with promotion and leaning toward greater visibility for the School's archives and collections for the sake of allowing its students (and teachers) to take greater advantage of them. Together with this pedagogical and didactic role, so fundamental for a fine arts school, an equally necessary perspective is offered, that of a shared center where the investigation and production of work is oriented toward any number of well-established lines of research. And of course, research is also encouraged into the many other areas that may come up in the future following the opening of what will be the Center for Art Archives and Collections (CAAC Cuenca - the same initials, but with expanded content and meaning). All kinds of new projects will focus on the current situation facing the contemporary arts. Ultimately, the tasks of exhibiting and disseminating these collections should be structured around a greater connection with the cultural life of the city, and with 
its specific needs when it comes to the experience of art.

Those of us who are involved in this project are entirely conscious of the impossibility (or at least the inopportune nature) of maintaining the museum model and making no changes to it. Whether the museum is big or small, the model was adopted from the European cultural situation more than 200 years ago. It then continued without any substantial transformations in order to accommodate modern art generally speaking, and only recently to accept other forms of contemporary art (photography, video, cinema, art installations, multimedia art). The challenge lies in making the most of our unique archives and collections (and managing their constant growth thanks to ongoing creative and production efforts). Once they are unveiled, the goal is to develop an interactive connection around them between the civic context and the space where they are located.

This is no easy task. Not for any art center, each of which is dependent on the range of its particular strengths and weaknesses. But the most important thing is to turn necessity into virtue, and to respond objectively to one's own situation. In terms of CAAC and Cuenca, our intention has to do with the perspective of the integration of the center within the framework of the expositive and cultural offerings found in this city, to whose history we are indebted. As indicated at the beginning of this presentation, a city cannot build its identity without a full set of cultural references that it is able to adopt and make its own. But a cultural center like the one presented here, a space for the research, production, exhibition and conservation of contemporary works of art, cannot help but to stretch itself to complement those references, filling in the gaps, and offering itself as a space for specific kinds of experiences. And that's exactly what we're working on.

\section{José Ramón Alcalá}

Vicente Jarque 
Detalle de la Sala Parkett renovada con motivo de la exposición "Nuevos

planteamientos de investigación en las CAAC. Pasado, presente y futuro" realizada

para las primeras jornadas oficiales de las CAAC. Comisarias: Beatriz Escribano y

Cristina Peña. Facultad de Bellas Artes. Cuenca. Abril 2016 / Detail of the Parkett

Room renewed for the exhibition "New research approaches in the CAAC. Past,

present and future" made on the occasion of the first official conference of the CAAC.

Curators: Beatriz Escribano y Cristina Peña. School of Fine Arts. Cuenca. April 2016
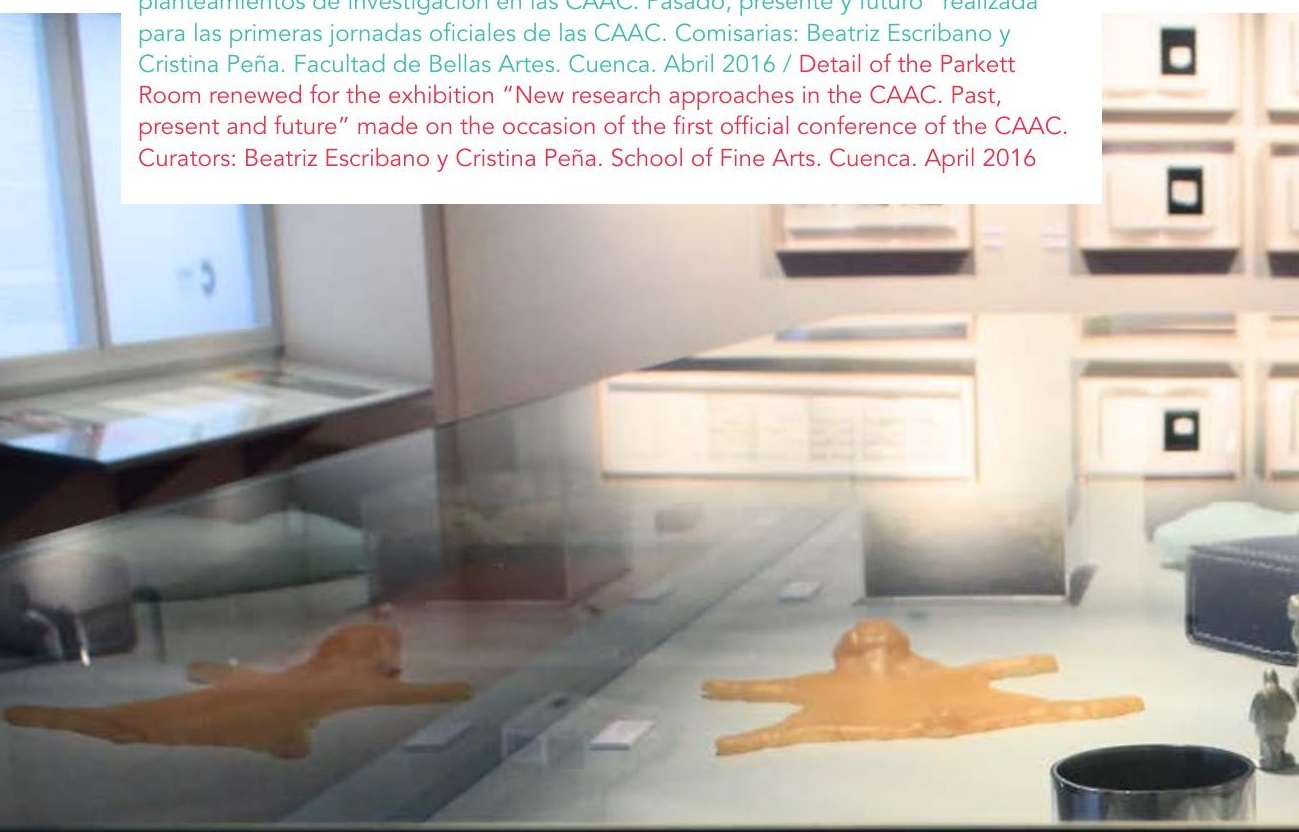

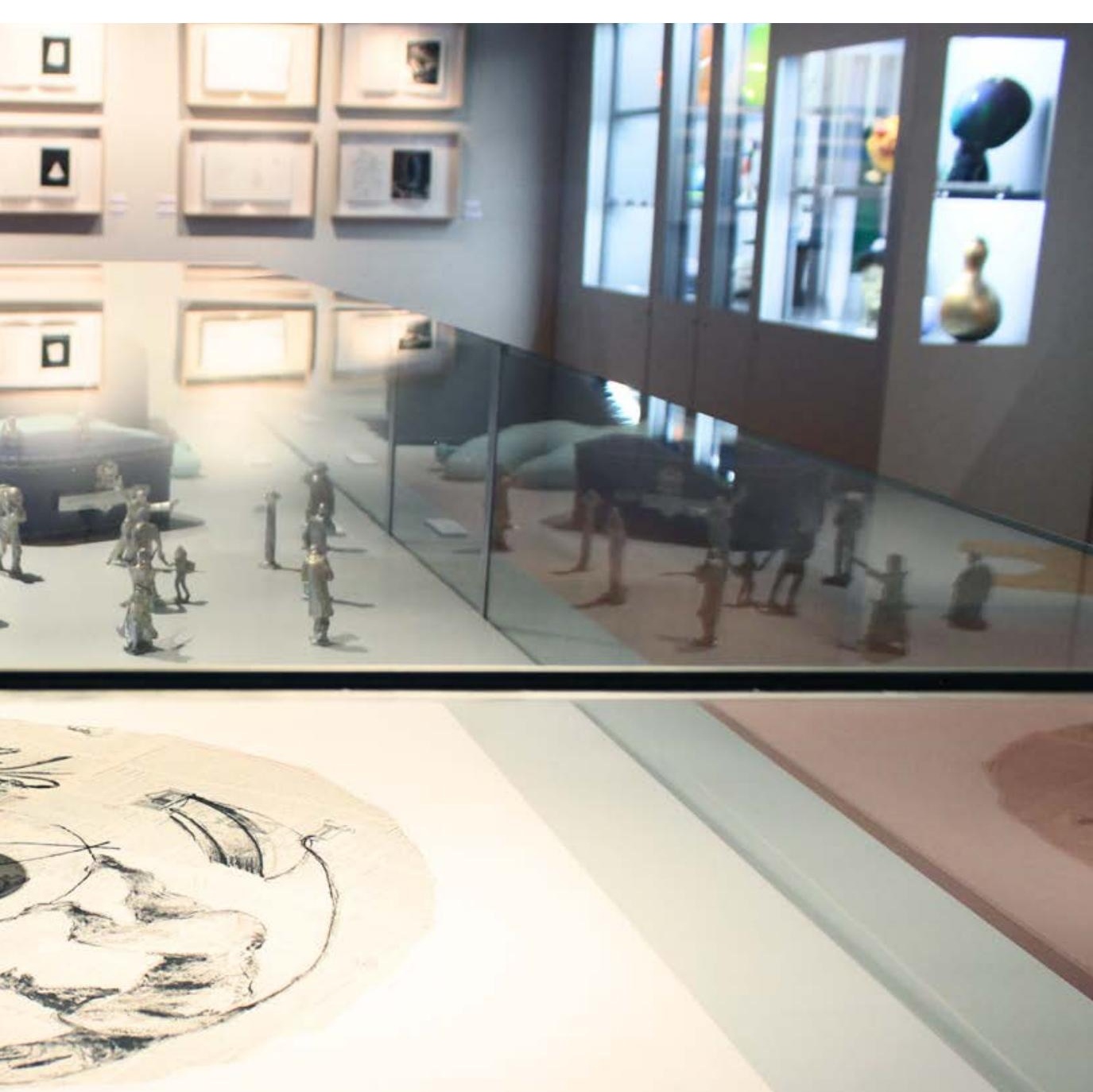
Detalle de la Sala Parkett renovada con motivo de la exposición "Nuevos

planteamientos de investigación en las CAAC. Pasado, presente y futuro" realizada

para las primeras jornadas oficiales de las CAAC. Comisarias: Beatriz Escribano y Cristina Peña. Facultad de Bellas Artes. Cuenca. Abril 2016 / Detail of the Parkett Room renewed for the exhibition "New research approaches in the CAAC. Past, present and future" made on the occasion of the first official conference of the CAAC.

Curators: Beatriz Escribano y Cristina Peña. School of Fine Arts. Cuenca. April 2016
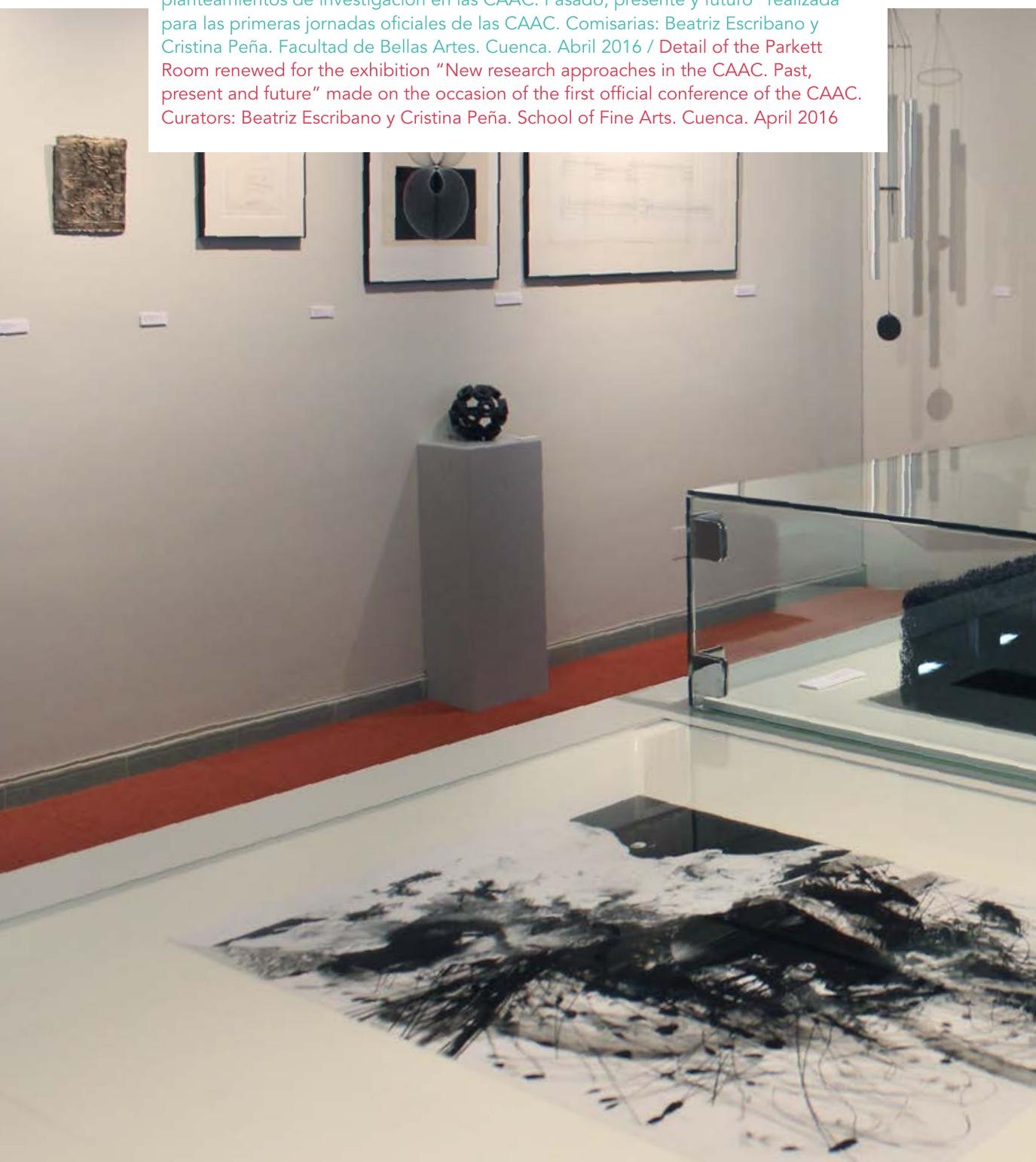


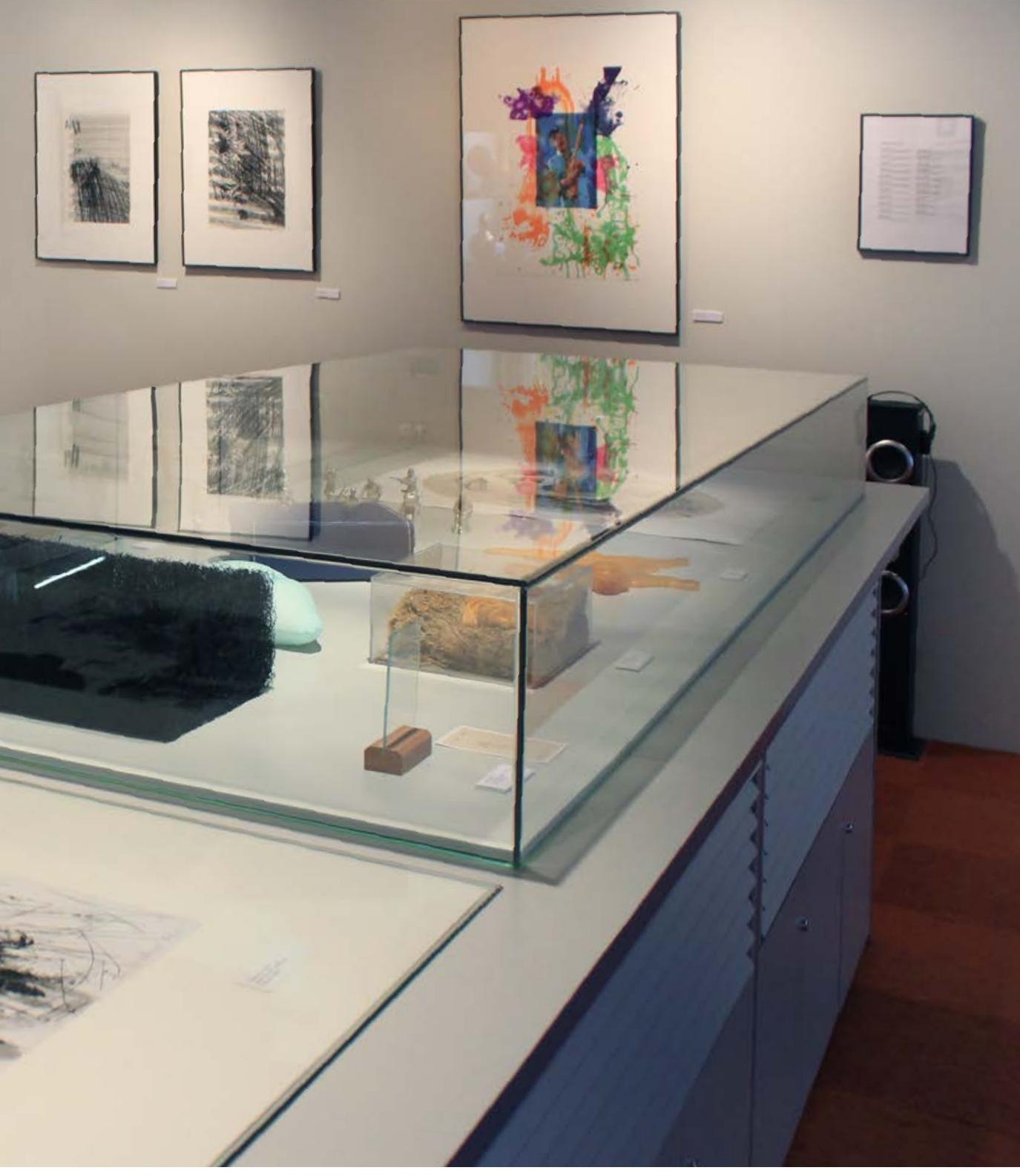






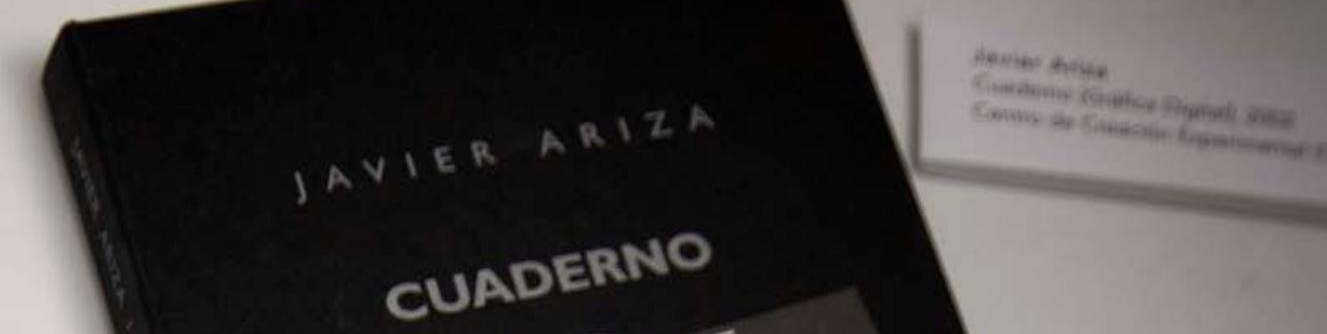

4
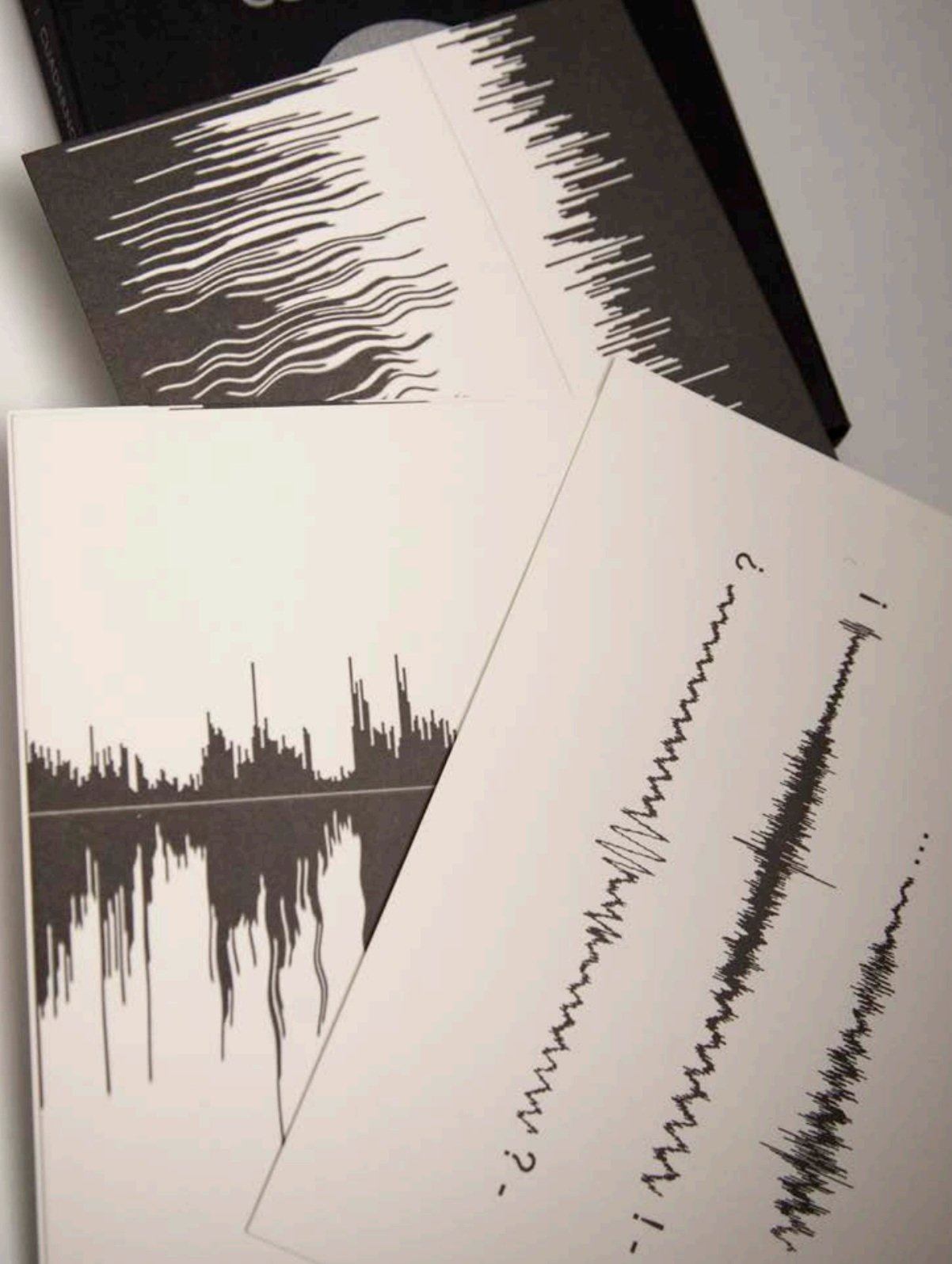


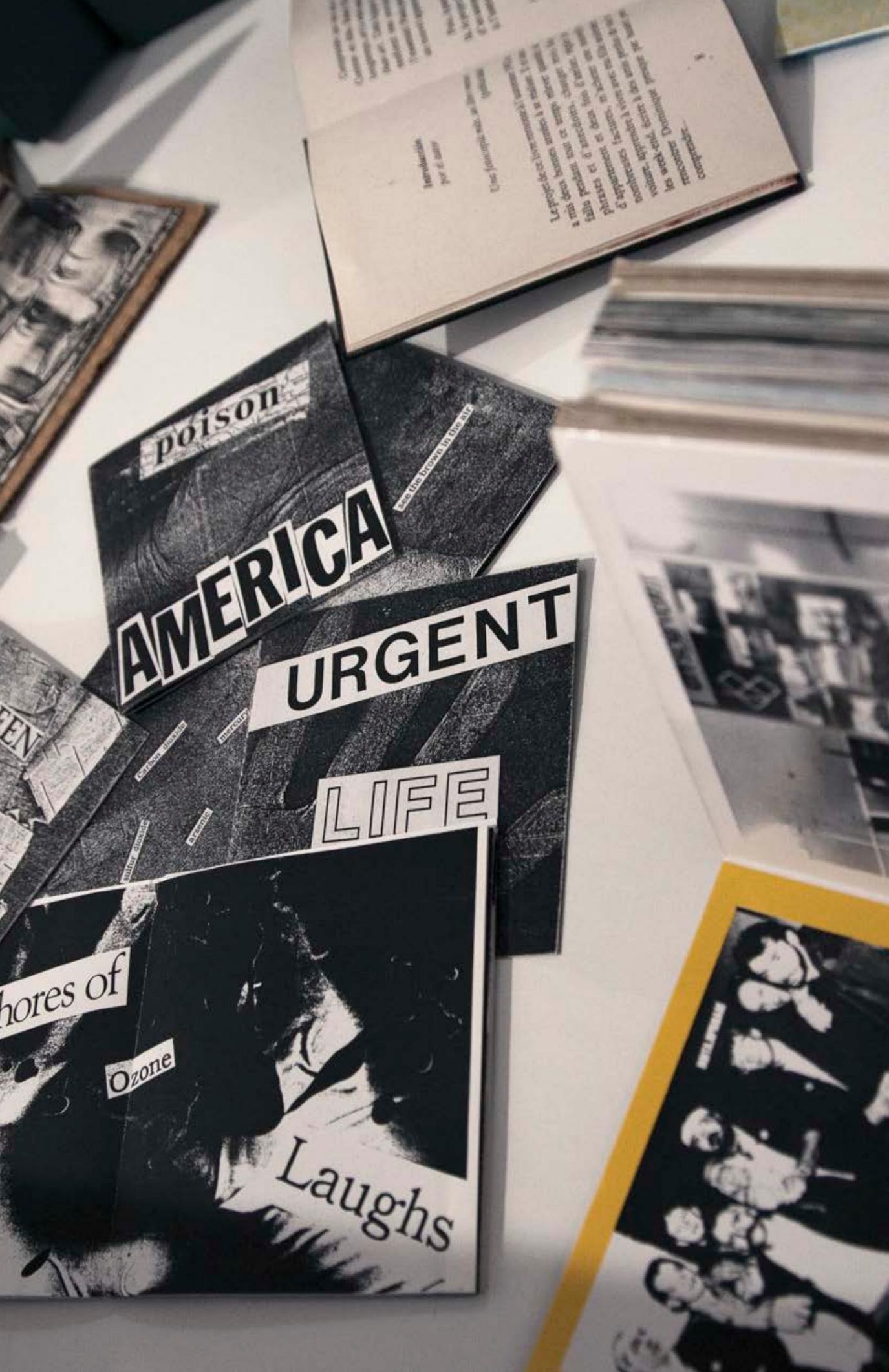


Visitas realiadas a los fondos CAAC por diversas instituciones educativas duante el período comprendido entre 2013 y 2016 / Visists to the CAAC founds by different educational institutions during the period between 2013 and 2016

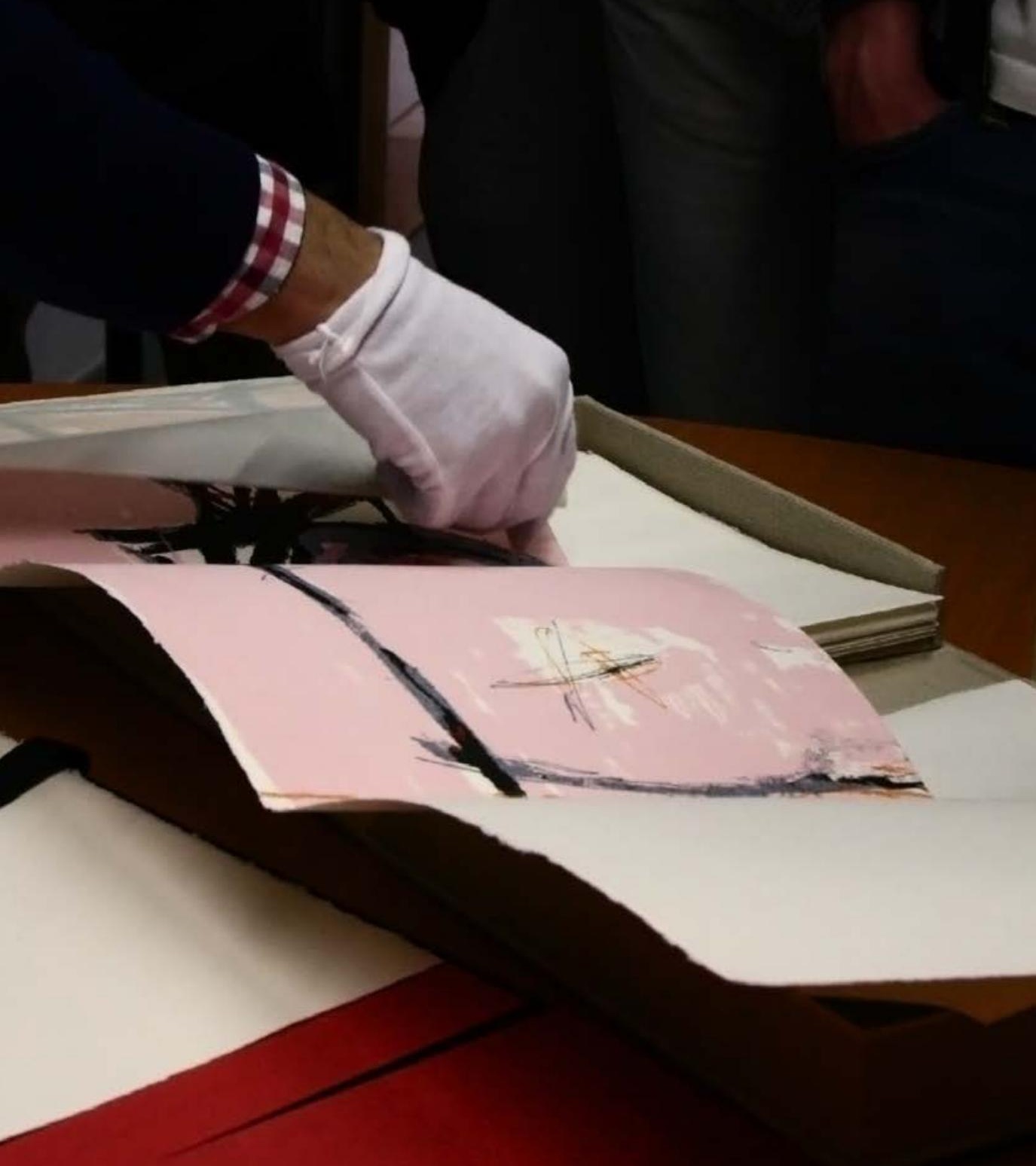




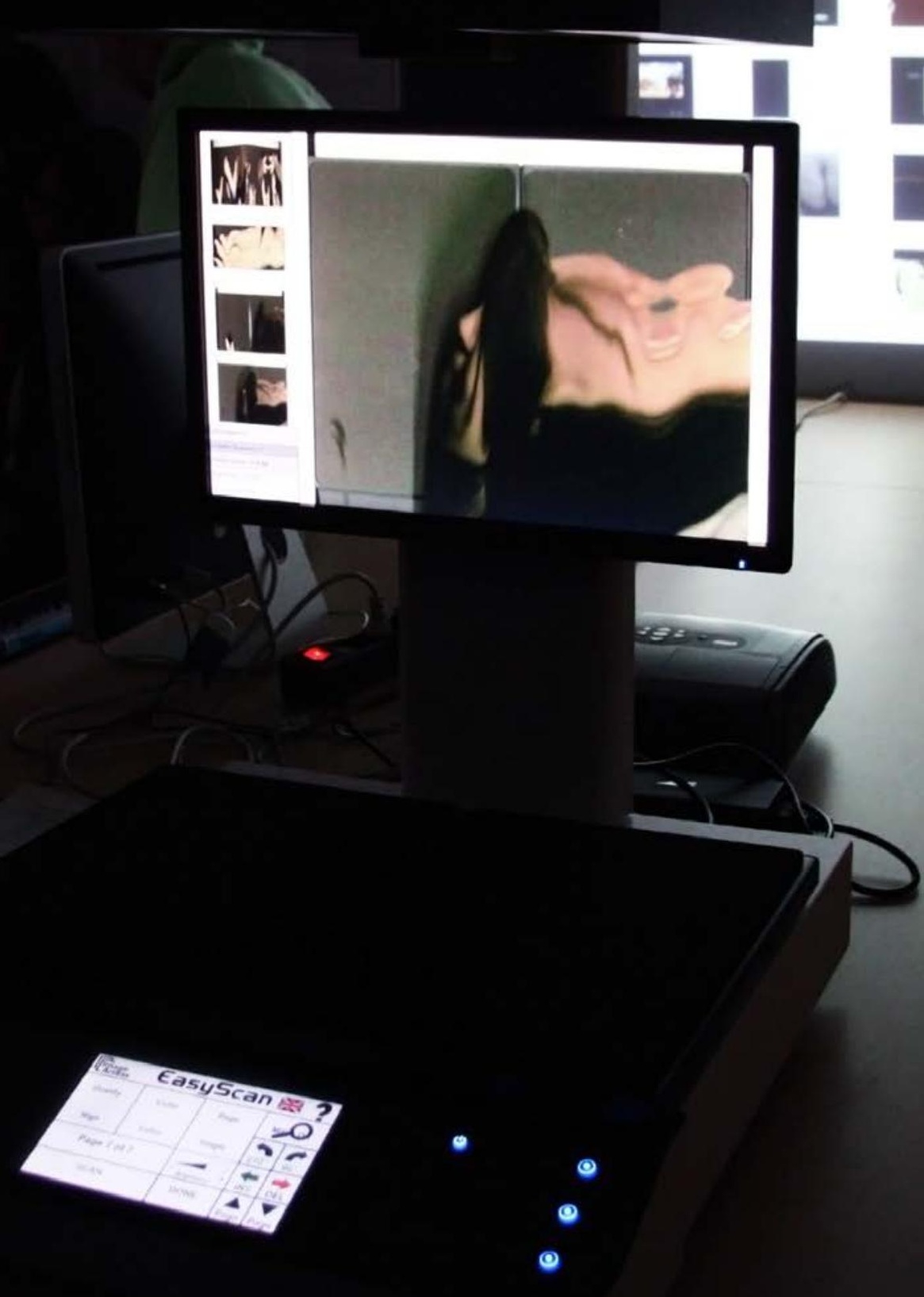


Exposición de los Fondos de Obra Gráfica de la Galería Juana Mordó y Medios de Impresión, realizada para las primeras jornadas oficiales de las CAAC. Comisario:

Ramón J. Freire. Sala ACUA UCLM. Cuenca. Abril 2016 / Exhibition of the Graphic Art Collection from the Juana Mordó Gallery and the Print Media Collection, made on the occasion of the first official conference of the CAAC. Curator: Ramón J. Freire. UCLM ACUA Room. Cuenca. April 2016.

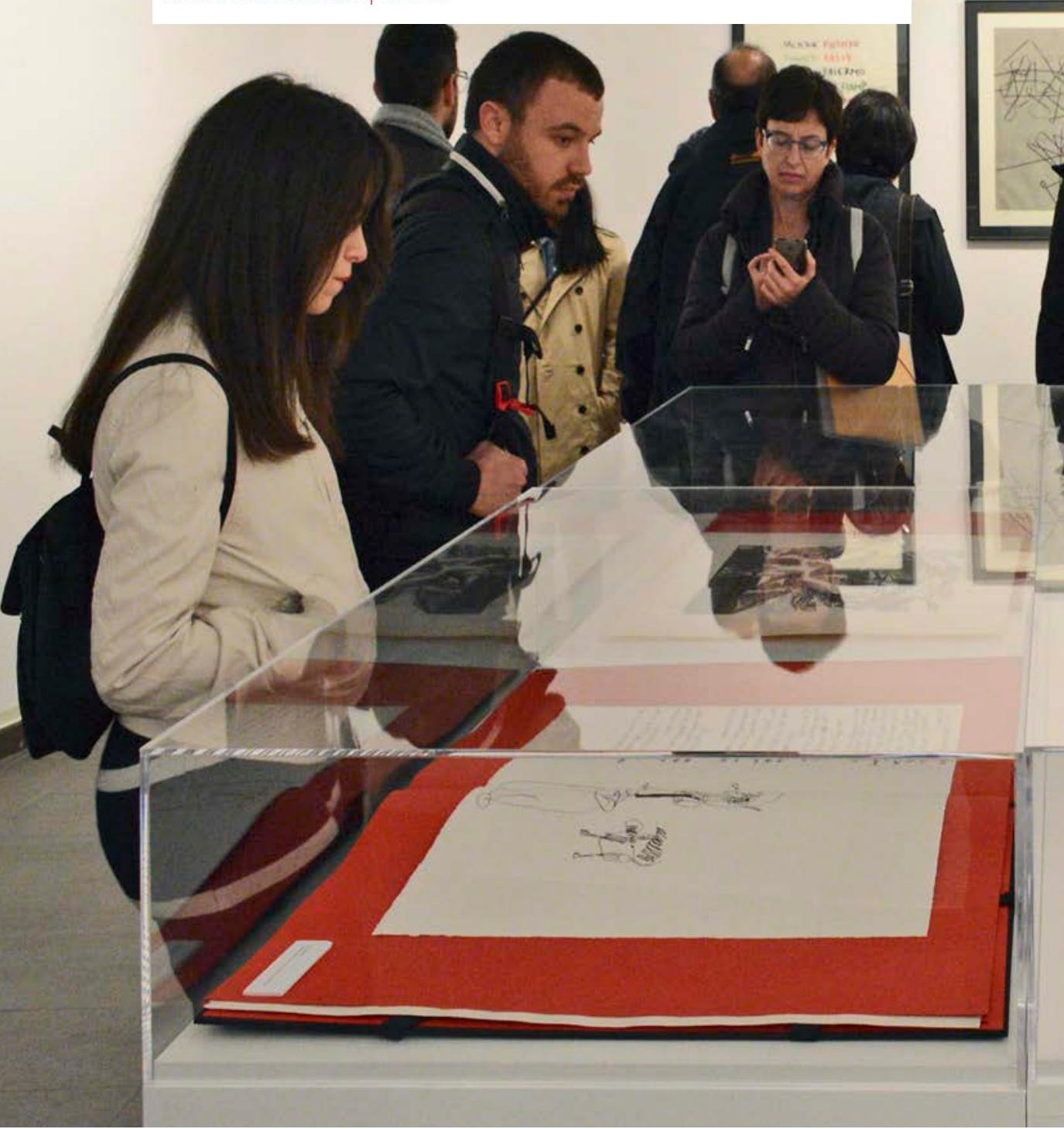




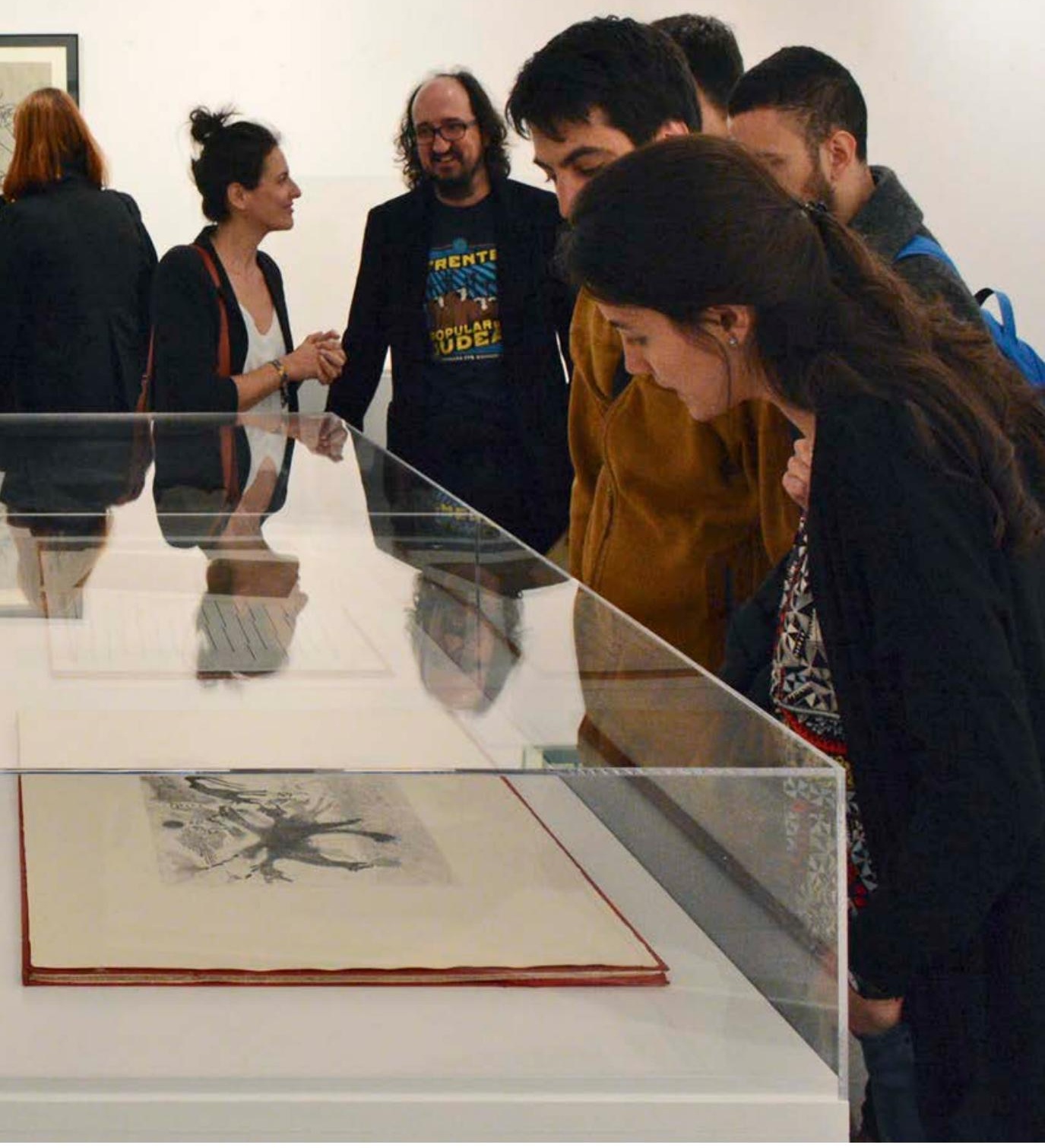





\section{Cuenca: desde el puente de los años}

E1 30 de junio de 1966 se abría el Museo de Arte Abstracto Español, situado en el centro histórico de Cuenca, ocupando algunas de las Casas Colgadas de la ciudad. Una aventura difícil de imaginar en un contexto cultural español que vivía ya la efervescencia de los cambios políticos y culturales, que cristalizarán formalmente con la aprobación de la nueva Constitución a mediados de diciembre de aquel mismo año. En ese contexto podemos situar las conversaciones tenidas en Cuenca en el verano de 1963 y en las que participan Gustavo Torner, Eusebio Sempere y Fernando Zóbel. La que inicialmente podría interpretarse como la historia de una amistad, pronto transcendería su espacio humano para imaginar desde él un proyecto que significaría para la historia del arte español de la segunda mitad del XX uno de sus capítulos más relevantes. No hay que olvidarse de que habrá que esperar dos largas décadas para que las dos principales instituciones dedicadas al Arte Moderno abran sus puertas: el IVAM y el Museo Reina Sofía lo harán en 1989, iniciándose desde entonces un largo proceso de proyectos varios que tejen la red de los museos que a lo largo de la geografía española se dedican actualmente al trabajo del Arte Moderno y Contemporáneo.

Volver hoy, 50 años después, a recordar aquellos años '60 supone un doble ejercicio de reflexión. Por una parte, la memoria de 50 años que partiendo de aquellas primeras decisiones dieron lugar a la creación del Museo de Arte Abstracto Español y toda su incidencia artística no solo en la ciudad de Cuenca sino en el marco del arte español. Una y otra vez se ha contado esa historia protagonizada desde su origen por los artistas que formaron el Grupo El Paso integrado por los ya citados Zóbel, Torner, Sempere a los que se sumarían Rivera, Millares, Saura, Canogar, Palazuelo, Feito, Viola, Chirino, Chillida, Tapies... cuya obra pasa a formar parte de las colecciones del Museo de Arte Abstracto Español. Por otra parte, la pregunta por imaginar 
una historia que recorra las próximas décadas y que partiendo de la experiencia artística de este pasado medio siglo se proyecte en nuevas ideas y trabajos. Esta reflexión debe articular los dos momentos citados, el de los años vividos y aquellos otros que se dibujan como horizonte en una perspectiva que hace suyos los grandes cambios de nuestra época.

Y si aquellos años '60 sintonizaron con lo que hoy podríamos llamar una forma de pensar en un contexto claramente internacional, hoy toda reflexión sobre el futuro debe hacer suyos los nuevos contextos y aquellas situaciones que definen la geografía del arte y la cultura contemporáneas. Cada vez resulta más necesaria una mirada hacia la década de los '60 para poder identificar las tensiones que han recorrido de forma trasversal los problemas de la cultura artística de nuestro tiempo. Fue entonces que emergieron con fuerza las preguntas relativas a la orientación de las sociedades contemporáneas, sus formas económicas, políticas, sociales y culturales. Fueron aquellos años un verdadero laboratorio en el que cristalizaron las formas y discursos de una nueva cultura que se instalaba en la frontera simbólica de lo que ya no era posible y de aquello otro que todavía no se había producido. Un doble trabajo, el de un duelo por la pérdida de un heroísmo que las Vanguardias habían reivindicado en su día para el arte y, por otro, el furioso experimento de las formas y los gestos que invadieron los diversos escenarios de aquellos años y que tienen en las Colecciones del Museo de Arte Abstracto Español un lugar relevante.

En efecto, una primera lectura nos permite entrar en el amplio debate de ideas que recorrió de una forma plural la civilización postindustrial, base de la actual. Frente a esta cuestión se afirmaban dos dispositivos complementarios; uno, dominado por la crítica de las formas y legitimaciones que acompañaban a la instrumentalización del Movimiento Moderno; otro, la búsqueda de nuevos procedimientos para constituir nuevos territorios sobre los que reinventar el orden 
de lo cotidiano. Tanto en uno como en otro caso coinciden ambos en llevar a cabo una abierta crítica de la ideología de la forma, de un positivismo de la función y la mecanización, causas principales de un proceso creciente de abstracción y homologación que hacía posible la eliminación de las condiciones humanas del proyecto.

Se trataba de una crítica que recorría por igual los planteamientos del arte y la arquitectura, situados ahora en una distancia crítica que interpelará por igual los principios del Movimiento Moderno y de las Vanguardias históricas, los nuevos humanismos o las ilusiones del socialismo utópico. Era necesario ir más allá de las confrontaciones convencionales y abrir la cultura del proyecto, entendida en su dimensión más amplia, a otros territorios, tal como los Situacionistas lo habían planteado a finales de los ‘50, exploración que podía tener en su forma radical la efímera poética de los gestos, pienso en Constant, por ejemplo.

Y si tanto el Expresionismo abstracto americano como el Informalismo europeo, de los que los artistas de El Paso son deudores, habían insistido en la crítica a la forma, el regreso a los materiales y al experimento de una escritura que se asomaba al vacío generando sobre él, gestualmente, los rastros, huellas de un tiempo que el arte interpretaba desde sus nuevas posiciones poéticas, aquellos años dieron lugar a un programa de discursos que permitieron construir un nuevo horizonte de cuestiones que a lo largo de las décadas sucesivas el arte explorará.

De manera generalizada comenzarán a emerger programas, situaciones que en su conjunto irán trazando un universo de signos en los que ya se anuncia el nuevo orden de ideas y mundos. Una tensión creativa que permitiría al arte reinventar sus relaciones con su época, al tiempo que prefigurar otro horizonte al que nosotros ya pertenecemos. La tensión utópica que había acompañado el movimiento de las Vanguardias, regresaba ahora en el contexto crítico y radical de 
quienes pensaban que el arte era el laboratorio privilegiado donde experimentar las formas culturales del futuro.

Si este análisis nos permite situar la lectura de lo acontecido a partir de los ‘60, marco de referencia de la creación del Museo de Arte Abstracto Español de Cuenca, una mirada sobre nuestro tiempo puede orientarnos mejor en la comprensión de la situación del arte en la cultura contemporánea. En las últimas décadas el arte ha jugado un papel creciente a la hora de establecer los códigos de lectura de todos aquellos problemas que, desde la identidad a la diferencia cultural, las formas del poder y su uso, emergencias y conflictos varios, han ido definiendo las transformaciones del mundo contemporáneo. $\mathrm{El}$ arte ha sido una de las instancias críticas más incisivas a la hora de explicar las tensiones simbólicas que configuran el horizonte antropológico de nuestro tiempo. Por otra parte, los procesos de globalización han roto las fronteras que delimitaban territorios acotados, dando lugar a una mundialización de problemas y lenguajes, favorecida por intereses nunca ajenos a la institución del arte. Este hecho nos permite situarnos hoy frente a una discusión abierta que no solo interprete, sino que problematice el alcance de la obra de arte en el contexto de la cultura contemporánea.

Si antes podíamos afirmar que resultaría difícil establecer la agenda de los problemas del mundo contemporáneo sin acudir al trabajo del arte como intérprete de estos, igualmente se puede decir que el arte ha sabido configurar un horizonte multicultural en el que las fronteras se reinterpretan y modulan de acuerdo a la nueva complejidad. Y es justamente el arte con sus lenguajes e intervenciones el que ayuda a construir una mirada abierta hacia una época compleja. Y si se habla hoy de una cultura de la post-identidad-Cultures In-between, dirá Bhabha - es para indicar los procesos de desplazamiento que descentran y permeabilizan los referentes tanto simbólicos como imaginarios de la cultura contemporánea.

Todos estos elementos son la base de un posible punto de partida 
para repensar el proyecto del arte en un contexto como el sugerido por la celebración del 50 aniversario de la creación del Museo de Arte Abstracto Español de Cuenca. La amplia y rica experiencia acumulada a lo largo de estos años - del festival de Música Religiosa al Gabinete de Música Electroacústica, las Fundaciones Antonio Saura y Antonio Pérez, la creación de la Facultad de Bellas Artes ya en 1985, la del MIDE, Museo Internacional de Electrografía, para terminar con momentos importantes de discusión y debate como La Situación, etc., sin olvidar las diferentes Colecciones que construyen por sí mismas una forma de la memoria del arte - hace que este sea el momento de poder construir sobre una base plural un proyecto de ideas e intervenciones con el correspondiente apoyo institucional. Siempre he pensado, siguiendo la lección de Max Weber, que las instituciones deben ser un puente entre los problemas y las ideas, deben ayudar a construir con sus mediaciones las condiciones exigidas por cada proyecto.

Lo importante ahora es construir una nueva forma de pensar, acorde con las condiciones de la nueva complejidad. Hoy, por ejemplo, la ecología nos obliga a pensar la ciencia y la política al mismo tiempo. Es la debilidad de ciertos discursos sobre la sostenibilidad que terminan siendo un inútil pliego de buenas intenciones. Si nos situamos en esa perspectiva, todo lo que tiene que ver con la cultura del proyecto debe ser repensado. John Berger lo recordaba recientemente. La primera tarea de toda cultura es proponer una comprensión del tiempo, de las relaciones del pasado con el futuro, entendidas en su tensión, en la dirección en la que convergen contradicciones y esperanzas, sueños y proyectos. Comme le rêve, le dessin! Sí, como el sueño, el proyecto en esa extraña relación en la que se encuentran las ideas y los hechos, la tensión de un afuera que la historia transforma y el lugar de un pensamiento que imagina y construye el mundo, la ciudad.

Y es Cuenca el lugar en el que este proyecto es pensado y ahora forma parte de un imaginario que lo sueña y espera construir. Vienen a 
la memoria las conversaciones entre Zóbel, Torner y Sempere allá por el verano de 1963, de ellas nació apenas tres años después el Museo de Arte Abstracto Español. Hoy es el momento de imaginar y construir un nuevo proyecto que desde la experiencia acumulada sea la referencia simbólica y operativa de todas aquellas tensiones, problemas e ideas que atraviesan nuestra época y buscan un lugar, una especie de laboratorio en el que hacer posibles nuevas formas de experiencia.

Francisco Jarauta

Cuenca: across the bridge of years

\section{Cuenca: across the bridge of years}


On June 30th, 1966, the Spanish Abstract Art Museum opened its doors. Located in Cuenca's old quarter, it was took up residence in the city's Hanging Houses. Such an adventure is hard to imagine given the Spanish cultural context which was already witnessing the effervescence of political and cultural changes that would be formally consolidated with the approval of the new Constitution in mid-December of that same year. It is against that background that we can place the conversations held in Cuenca in the summer of 1963 involving Gustavo Torner, Eusebio Sempere and Fernando Zobel. What might at first glance be interpreted as a story of friendship would quickly transcend human space giving way to a project that would signify one of the most relevant chapters for the history of Spanish art in the second half of the 20th century. We mustn't forget that it would still be two long decades before the two main Spanish institutions dedicated to Modern Art would open their doors. The IVAM and the Reina Sofía Museum would open in 1989, beginning the long process of weaving together the network of museums throughout Spain which are currently dedicated to the work of Modern and Contemporary Art.

Looking back now, 50 years later, and remembering those years in the " 60 s is a double exercise in reflection. All of the memories of the 50 years that followed those first decisions led to the creation of the Spanish Abstract Art Museum, and all of the artistic effects not only in the city of Cuenca, but in all of Spanish art. Time and again, we have heard that story starring the artists who made up the El Paso Group, whose members included Zobel, Torner and Sempere, as we have mentioned. They would be joined by Rivera, Millares, Saura, Canogar, Palazuelo, Feito, Viola, Chirino, Chillida, Tapies... whose work would become part of the permanent collection at the Spanish Abstract Art Museum. And looking forward, we must imagine a history that carves out the coming decades, and which, inspired by the artistic experience of the last half-century, leads to new ideas and 
projects. This reflection must focus in both directions, that of years past, and that of others which are sketched like the horizon on a perspective that claims ownership of the great changes of our age.

And if the 1960s were calibrated with what today we might call a way of thinking in a context that is clearly international, these days all reflections on the future must claim new contexts and those situations that define the geography of art and contemporary culture. It is increasingly necessary to look back on the 1960s in order to identify the tensions that run through the problems of artistic culture in our day. It was then that the relative questions sprung forth as to the orientation of contemporary societies, and their economic, political, social and cultural forms. Those years were a veritable laboratory for the crystallization of form and discourse for a new culture that was taking shape on the symbolic frontier between what was no longer possible and what had still not been produced. A double project, that of a duel for the loss of a true hero that the vanguard had claimed for art in its own time, and of the furious experiment of shapes and gestures that invaded various venues in those years, and that play a prominent role in the permanent collection at the Spanish Museum of Abstract Art.

In fact, a first reading allows us to engage in the broad debate of ideas that explored in a pluralistic manner the post-industrial civilization which is the basis for our current society. Faced with this question, two complementary devices were confirmed: one, dominated by the criticism of the forms and entitlements that were part and parcel of the manipulation of the Modern Movement; and another, involving the search for new procedures in order to constitute new terrain where a new daily order could be re-invented. In both cases, an open criticism is carried out as to the ideology of form, of a positivism surrounding function and mechanization, the principle causes of a growing process of abstraction and accreditation that made possible 
the elimination of human conditions from the project.

This was a critique that explored in equal measure the approaches of art and architecture, now situated at a critical distance that will question equally the beginnings of the Modern Movement, historical vanguard, new humanisms, and the illusions of utopic socialism. It was necessary to go beyond conventional confrontations and expand to other territories the project's culture, understood in its broadest sense. This is what the Situationists took on in the late 1950s, an exploration whose most radical form included the ephemeral poetics of the gesture. The example of Constant comes to mind.

And if both American Abstract Expressionism and European Informalism (to which the E1 Paso artists are indebted) had insisted on a criticism of form, a return to materials and an experience of writing that leaned out into the void, thus generating above it, gesturally, the features, the marks of a time interpreted by art from its new poetic position, those years led to a discursive program that allowed for the construction of a new horizon of questioning that would be explored by art throughout the coming decades.

Generally speaking, the emergence would begin of programs, situations that, taken as a whole, would trace out a universe of symbols in which a new order of ideas and worlds can already be seen. A creative tension that would allow art to re-invent its relationship to its time, while foreshadowing another horizon, the one to which we belong. The utopic tension that had accompanied the avant-garde movement now made its return in the critical and radical context of those who thought of art as a privileged laboratory for experiencing the cultural forms of the future.

If this analysis allows us to situate a reading of that which occurred following the "60s, giving us a frame of reference for the creation of the Spanish Abstract Art Museum in Cuenca, a look at our time might 
do a better job of helping us to understand the placement of art in contemporary culture. In the last few decades, art has played an increasingly important role in establishing codes for reading those problems which have defined the transformations of the contemporary world, from the identity of cultural differences, to various forms of power and its use, emergencies and various types of conflict. Art has been one of the most assertive critical instances when explaining the symbolic tensions that make up the anthropological horizon of our day. As a matter of fact, the process of globalization has broken the borders that once defined the limits between territories, leading to a globalization of problems and languages, favored by interests that are never divorced from the institution of art. Today, this fact permits us to join an open discussion that not only interprets the reach of artwork in the context of contemporary culture, but which leads to problematization.

Before, it may have been difficult to establish the agenda of the contemporary world's problems without turning to the work art does as an interpreter of maladies, but we could also say that art has managed to put together a multicultural horizon where borders are re-interpreted and modeled in accordance with a new sort of complexity. And it is precisely art, with its languages and interventions, that helps to build an open view toward a complex era. And if today we speak of a culture of post-identity (what Bhabha would call culture's 'in-between'), it is in order to indicate the processes of displacement that de-center and permeate contemporary cultural reference points that are both symbolic and imaginary.

All of these elements are the basis for a possible guiding principle for rethinking the project of art in a context like the one suggested by the 50-year anniversary of the creation of the Spanish Abstract Art Museum in Cuenca. The diverse and meaningful experience gathered over the years -the Religious Music Festival, the Electronic $\mathrm{Mu}-$ sic Course, the Antonio Saura and Antonio Pérez Foundations, the 
creation of the School of Fine Arts in 1985, the creation of MIDE, the International Museum of Electrography, and significant moments of discussion and debate like The Situation, for example, and of course various collections that create for themselves a sort of artistic memory - all of these make this the moment for building atop a multi-pronged foundation a project composed of ideas and interventions that is duly held up by institutional support. I have always believed, following the lessons of Max Weber, that institutions must serve as bridges between problems and ideas, helping to create the conditions required by each project through their intermediary efforts.

The most important thing now is to build a new way of thinking, in keeping with the conditions of a new complexity. Today, for example, ecology makes it necessary to consider science and politics simultaneously. This is the weak point of certain speeches on sustainability which end up as useless piles of good intentions. If this is the perspective we take, everything having to do with the culture of the project must be re-thought. John Berger recently recalled this point. The first task of every culture is to propose a way of understanding time, of relating past events with the future, understood in their tension, in the direction in which contradiction and hope, dreams and projects, converge. Comme le rêve, le dessin! Yes, like the dream, the project is like that strange relationship where we find ideas and deeds, the tension of an outside element which is transformed by history, and the space for thinking that imagines and builds the world, the city.

And Cuenca is the place where this project was conceived, and which now forms part of the imagery it dreams of and hopes to build. Our memory is filled with conversations between Zobel, Torner and Sempere in the summer of 1963, the conversations that would lead to the opening of the Spanish Abstract Art Museum just three years later. Now is the time for imagining and building a new project which, drawing on gathered experience, will be the symbolic and operative 
Cuenca: across the bridge of years

reference point for all those tensions, problems and ideas that are passing through our era, looking for a place, a sort of laboratory where they can create new sorts of experiences.

Francisco Jarauta 

Jornada de puertas abiertas de las CAAC, dentro del programa "MICRO-CAAC:

Pequeñas actividades, grandes acciones", celebradas el 20 de Marzo de 2015 /

CAAC's Open day, within the "MICRO-CAAC: Small activities, great actions" program, held on March 20. 2015.

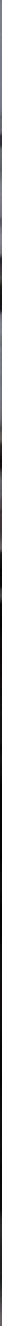




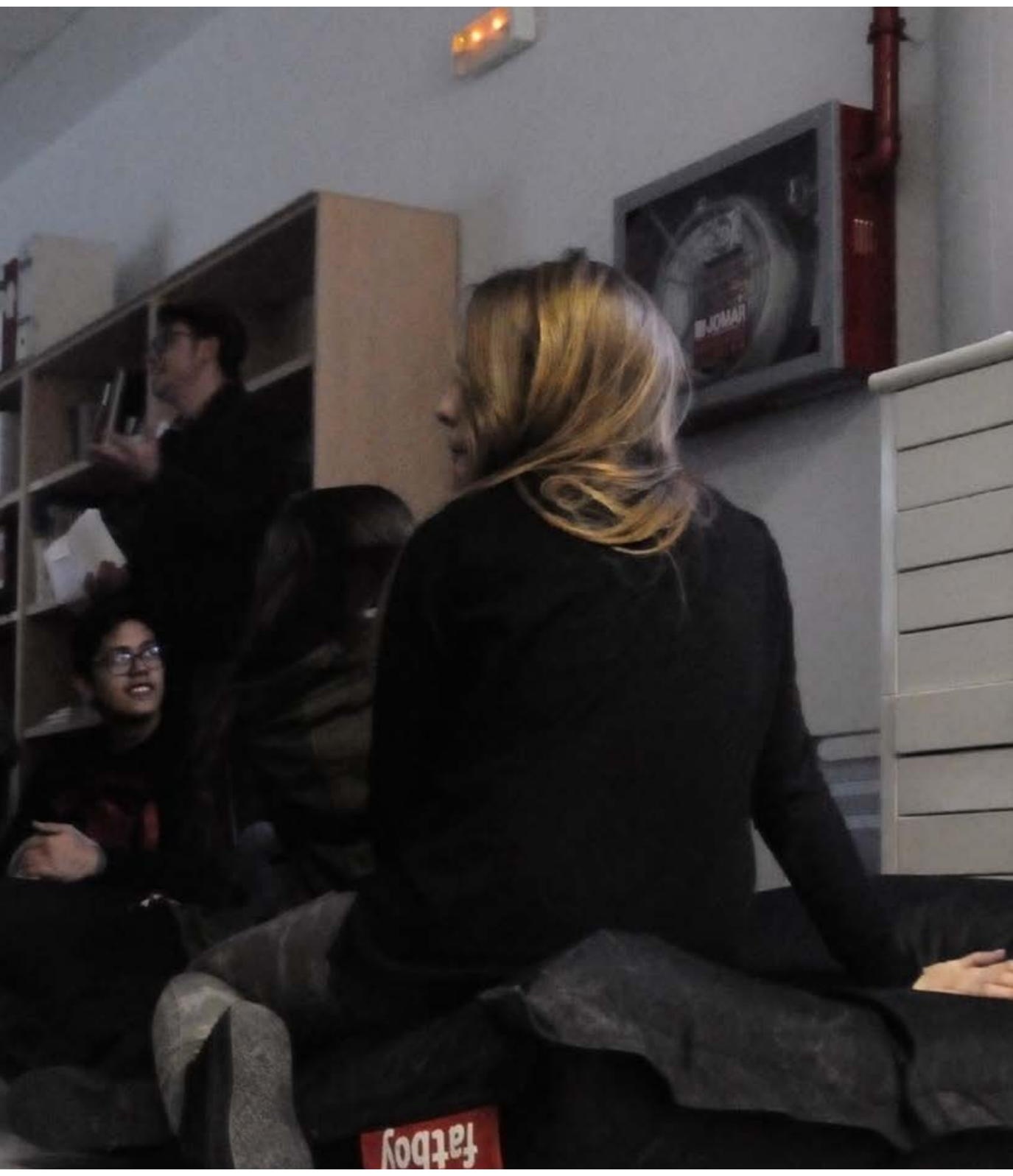


Mesa de trabajo celebrada durante las primeras jornadas oficiales de las CAAC

"Cuenca y sus Colecciones y Archivos de Arte Contemporáneo. Las CAAC como modelo patrimonial y de identidad ciudadana". Cuenca. Abril 2016 / Worktable held during the fist official conference of the CAAC "Cuenca and its Collections and Archives of Contemporary Art. The CAAC as an heritage model and civic identity". Cuenca. April 2016.

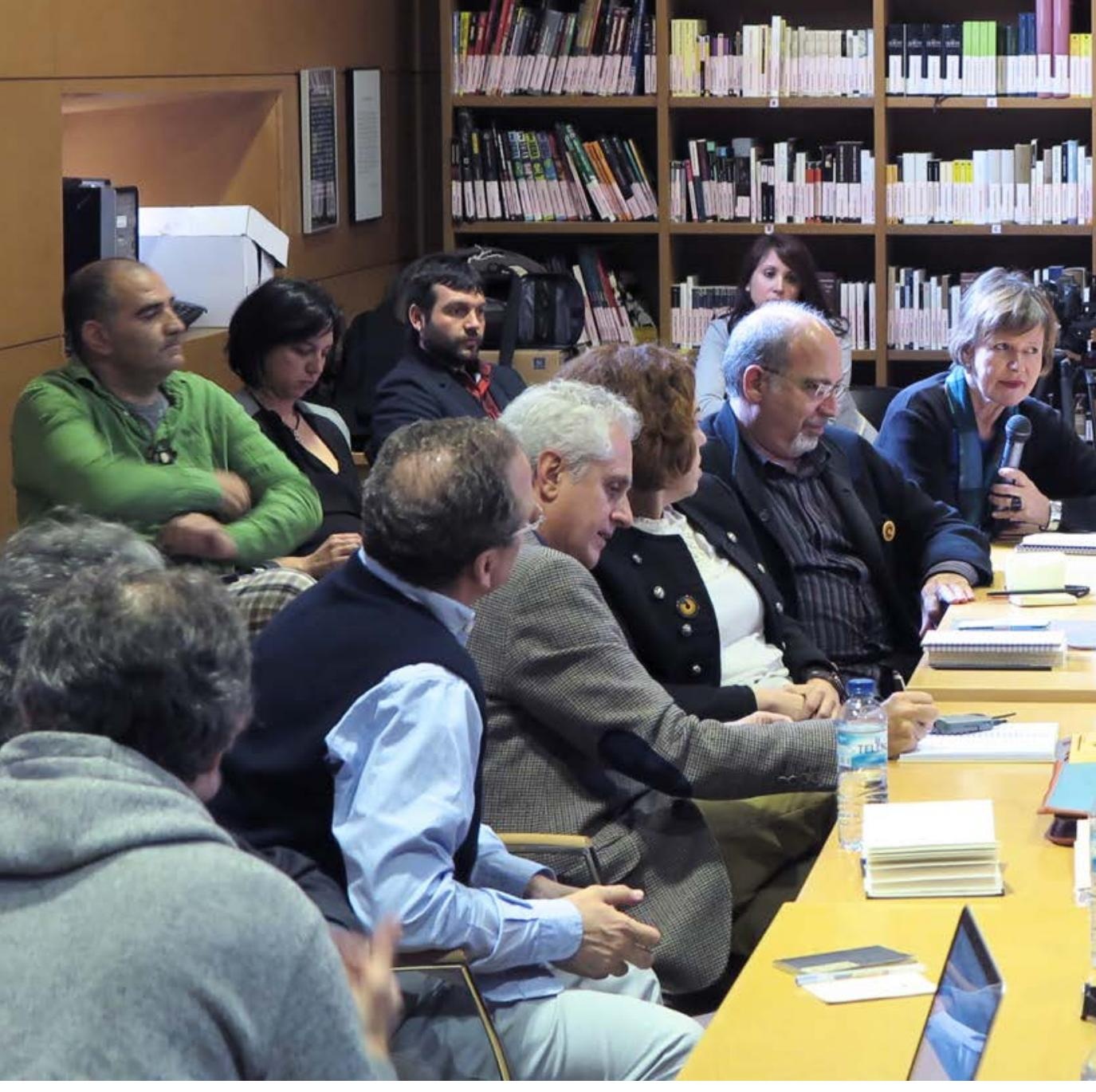




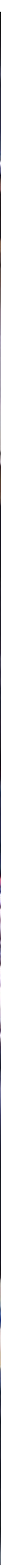


